\title{
Quasi-Genericity of Bifurcations to High Dimensional Invariant Tori for Maps
}

\author{
G. Iooss and J. E. Los* \\ Laboratoire de Mathématiques, U.A. CNRS 168, Université de Nice, Parc Valrose, \\ F-06034 Nice, France
}

\begin{abstract}
We consider a family of maps in a Banach space $E$ near the situation when the derivative at the fixed point has two pairs of complex eigenvalues lying on the unit circle, the other part of the spectrum being strictly inside the unit disc. We focus our attention on the region of the parameter space where the truncated normal form of the maps shows a bifurcation of a family of invariant $T^{1}$-circles into a family of invariant $T^{2}$-tori. We show that this problem needs a 3 dimensional parameter unfolding and that, for the complete maps, bifurcation occurs at points $\gamma_{\omega, \Omega}$, where $\omega$ is the rotation number on the non-normally hyperbolic $T^{1}$-circle, and $e^{ \pm 2 i \pi \Omega}$ are the eigenvalues of the constant matrix conjugated to the non-contracting part of the linearization on the normal fiber bundle over $T^{1}$. Making some non-resonance and diophantine assumptions on $(\omega, \Omega)$ leading to a positive measure Cantor set in $T^{2}$, we show that in paraboloidal regions of the $3 \mathrm{dim}$. parameter space we have "clean" bifurcations as for the truncated normal form. The complement of these regions forms a set of bubbles such as the ones obtained by Chenciner in [Chen] for a codimension 2 problem for maps in $\mathbb{R}^{2}$. The main tool here is a generalization for a matrix function on $T^{1}$, close to a constant, of the quasiconjugacy to a constant, modulo a minimum of additional parameters ("moved" quasi-conjugacy). For the infinite dimensional case we use a $C^{\infty}$ decoupling result on the angular dependent linear parts into a contraction, still angular dependent, and another part quasi-conjugated to a constant matrix. This type of analysis applies for a wide range of problems, where truncated normal forms of the maps give bifurcations from $T^{n}$ to $T^{n+1}$ tori, and this needs a $(n+1)$-dimensional parameter unfolding.
\end{abstract}

\footnotetext{
* We gratefully acknowledge the DRET (contrat 86/1445) who supported one of the authors (J.L.) during this work. This research has been also supported by the E.E.C. contract No. ST 2J-0316-C (EDB) on Mathematical problems in nonlinear Mechanics
} 


\section{Contents}

1. Introduction . . . . . . . . . . . . . . . . . . . . . . . . . . . . . . 454

2. Position of the Problem . . . . . . . . . . . . . . . . . . . . . 456

3. Hopf-Hopf Interaction for Maps . . . . . . . . . . . . . . . . . . . . . . . 458

4. Persistence of an Invariant Curve for Maps in $\mathbb{R}^{4}$. . . . . . . . . . . . . . . . . 464

5. Bifurcation of Invariant $\mathbb{T}^{2}$-Tori. Bubble Structure. . . . . . . . . . . . . . . . 477

6. Persistence and Bifurcation of Tori in Higher Dimensions. . . . . . . . . . . . . 483

Appendix: The Quasi-Conjugacy Problem. . . . . . . . . . . . . . . . . . . . 489

References . . . . . . . . . . . . . . . . . . . . . . 498

\section{Introduction}

Bifurcations of invariant tori of dimension larger than one for families of maps, or larger than two for families of vector fields in dissipative systems is a puzzling problem. There are experimental evidences of the existence of such tori, for instance in the Bénard convection problem [Go-Be] and in the Taylor Couette problem [GRS]. First mathematical attempts to study such bifurcations appeared in the papers by Sell [Sell] and Chenciner and Iooss [Ch-Io], where under very strong assumptions, they prove the bifurcation result. Weakest assumptions [Ch-Io] were mainly that there exists a $\mathbb{T}^{n}$-torus invariant under the mapping at criticality (non-normal hyperbolicity), where the non-contracting part of the linearization on the normal fiber bundle (over the torus) is conjugated to a constant matrix with eigenvalues of modulus one. Adding more assumptions on the rotation vector of the map along the invariant torus and on the arguments of the eigenvalues of the constant matrix, they proved that, under a transversality condition in the parameter space, a family of $\mathbb{T}^{2}$-tori persists near criticality and a family of invariant $\mathbb{T}^{n+1}$-tori bifurcates. All those assumptions made people think that these bifurcations are infinite codimension phenomena and then occurs rarely!

Another way to obtain high dimensional invariant tori is to study high codimension singularities. For instance, if we consider the very popular codimension 2 problem of a two parameter family of vector fields with a fixed point at the origin, such that at criticality, two pairs of complex conjugate eigenvalues are on the imaginary axis (Hopf-Hopf interaction), then under non-strong resonance conditions ( $[\mathrm{Gu}-\mathrm{Ho}]$ Sect. 7.5, [Io-La], [Io 81]) one can easily show, depending on some coefficients, that invariant $\mathbb{T}^{3}$-tori can bifurcate from the secondary branch of invariant $\mathbb{T}^{2}$-tori. In fact, this bifurcation is only clear on the truncated normal form of the vector fields. If we consider the true vector field family (a generic one), one could only prove that the $\mathbb{T}^{2}$-tori and $\mathbb{T}^{3}$-tori families exist outside of a little neighborhood (called a "black hole" on [Io 81]) in the parameter space, of the set where precisely bifurcation occurs for the normal form. This is due to the lack of normal hyperbolicity in this neighborhood as in [Chen], [Los 1].

In this paper, we show that such codimension 2 problems have to be unfolded, using an additional parameter, in the neighborhood of the bifurcation manifold. In this three dimensional parameter space, there is now a Cantor set of points where the ideal situation (assumed in [Ch-Io]) occurs. This leads to a Cantor set of little paraboloidal regions where we know what happens for the dynamics (Fig. 1), and a complementary region ("bubbles") where we don't know yet. 


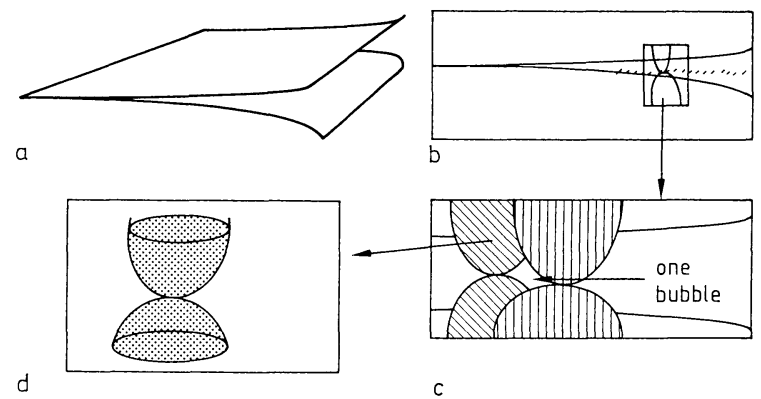

Fig. 1a-d. The parameter space with the Cantor set of paraboloidal regions and the bubbles. a The non-normal hyperbolicity region in the 3 dimensional space b. Section view $\mathbf{c}$. Paraboloidal region and one bubble in the plane section $\mathbf{d}$. One paraboloidal region in the 3 dimensional parameter space

A strong analogue of such a situation with one parameter less, was obtained by Chenciner [Chen] in the neighborhood of a degenerate Hopf bifurcation for maps, where such bubbles and parabolic regions in the parameter plane occur, near the circle saddle-node bifurcation curve, obtained with the normal form. In the same spirit, Los [Los 1,2] obtained also bubbles and parabolic regions in a codimension 2 problem involving a doubling of invariant $\mathbb{T}^{1}$-tori. A similar phenomenon was also obtained by Braaksma and Broer [Bra-B] in the study of a quasi-periodically forced Hopf bifurcation, leading to a bifurcation $\mathbb{T}^{n} \rightarrow \mathbb{T}^{n+1}$. Their study is however simplified by the fact that the flows on the $\mathbb{T}^{n}$-tori are linear in such a problem while this is not the case in our study for generic families. Another result of the same type was obtained by Scheurle [Sch] in the study of reversible vector fields where he shows how high dimensional invariant tori bifurcate.

The techniques developed below are directly adapted from the one used by Herman [Her] in his proof of the invariant curve theorem. This one is the main tool in the works of Chenciner [Chen] and Los [Los 1] for bifurcation problems. Namely, to prove the persistence of $\mathbb{T}^{1}$-tori, we extensively use the strong implicit function theorem in Fréchet spaces in the version of Hamilton [Ham]. The translated curve theorem of Rüssman [Russ] in the Herman formulation [Her], [Bost], or its adaptations [Chen], [Los 1] are now completed with a quasiconjugacy theorem for matrices near a constant one, generalizing a result obtained on a particular case in [Los 2]. An analogous result, for vector fields and in the case when matrices are close to a diagonal one with simple eigenvalues on the imaginary axis, is obtained by Moser [Mos]. He was the first to show the necessity of adding parameters to obtain the quasi-conjugacy to a constant. Our result gives the exact number of additional parameters, necessary in any case for the quasiconjugacy and this allows our system to satisfy the conditions described in [Ch-Io]. Finally the bifurcation result follows quite easily from the [Ch-Io] results, using these additional parameters defining new parameter space coordinates. The bifurcation to $n$-dimensional invariant tori occurs generically on a Cantor set which is defined by an $n$ parameters set having an $n$-dimensional positive measure. So we say that in some sense it is "quasi-generic." 


\section{Position of the Problem}

\subsection{Center Manifold and Normal Form}

To motivate our study, let us show several so-called codimension 2 singularities where we obtain a situation on which the techniques developed in this paper directly applies. We restrict our examples to families of maps, since analogous techniques could as well be adapted for singularities of vector fields (like the HopfHopf interaction).

Let us consider a family of maps in a Banach space $E$ :

$$
Z \rightarrow Z^{\prime}=\mathscr{F}_{\mu}(Z), \quad \mu \in \mathbb{R}^{k}, Z \in E,
$$

where $\mathscr{F}$ is $C^{\infty}$ in a neighborhood of 0 , and: $\mathscr{F}_{0}(0)=0$ and $D_{Z} \mathscr{F}_{0}(0)=\mathscr{T}_{0}$ is such that $\mathscr{T}_{0}$ has a spectrum separated into one part $\sigma_{-}$, inside the open unit disc in the complex plane, and another part $\sigma_{0}$ on the unit circle, which is assumed to be a finite union of isolated eigenvalues of finite multiplicities. The space $E$ is then decomposed as follows [Kato]:

$$
E=E_{0} \oplus E_{-},
$$

where the projections $P_{0}, P_{-}$respectively on $E_{0}$ and $E_{-}$commute with $\mathscr{T}_{0}$, and the restriction $T_{0,-}$ of $\mathscr{T}_{0}$ on $E_{0,-}$ has exactly the spectrum $\sigma_{0,-}$.

A first very useful result is the center manifold theorem (see for instance [Ma-Cr], [Io 79]), which says that all the dynamics of the iterates of $\mathscr{F}_{\mu}$ is locally attracted towards an invariant manifold $\mathscr{M}_{\mu}$ of class $C^{k}$ (for any fixed $k, \mathscr{M}_{\mu}$ exists in some neighborhood of 0 ) described as follows:

$$
\text { where } \left.\begin{array}{l}
Z=X+\Phi_{\mu}(X) ; X \in E_{0}, \\
\Phi_{0}(0)=0 \text { and } D_{X} \Phi_{0}(0)=0 .
\end{array}\right\}
$$

We might build $\Phi_{\mu}$ in $E_{-}$, but it is much better to be free to allow a part of $\Phi_{\mu}$ in $E_{0}$, since this allows us to choose the nicest possible coordinates in $E_{0}$ to obtain the trace of the map $\mathscr{F}_{\mu}$ on $\mathscr{M}_{\mu}$ written in normal form. Since it is clear that the interesting dynamics lie on a center manifold, we need to study such a normal form. The very simple result shown in [Io 81], see also [Bel], is that we can find $\Phi$ such that the map on $\mathscr{M}_{\mu}$ takes the form:

$$
\left.\begin{array}{c}
X \rightarrow X^{\prime}=F_{\mu}(X)=T_{0} X+N_{\mu}(X)+O\left(\|X\|^{P}\right) \\
N_{0}(0)=0 ; D_{X} N_{0}(0)=0 ; P \text { is arbitrary and }
\end{array}\right\}
$$

$T_{0}^{*}$ being the adjoint of $T_{0}$ in the finite dimensional space $E_{0}$.

\subsection{Hopf-Saddle-Node Interaction}

Here we assume $\sigma_{0}=\{1\} U\left\{\lambda_{0}\right\} U\left\{\bar{\lambda}_{0}\right\}$, where $\lambda_{0}=e^{2 i \pi \omega_{0}}$; all these eigenvalues being simple, we write:

$$
X=x \cdot \xi_{0}+z \cdot \zeta_{0}+\bar{z} \cdot \bar{\zeta}_{0} \in E_{0}
$$


where $\xi_{0}, \zeta_{0}$, and $\bar{\zeta}_{0}$ are the eigenvectors of $T_{0}$ belonging respectively to the eigenvalues $1, \lambda_{0}$ and $\bar{\lambda}_{0}$. We consider the map in $\mathbb{R} \times \mathbb{C}:(x, z) \rightarrow\left(x^{\prime}, z^{\prime}\right)$. Let us make a little remark here due to the imposibility to check numerically or physically whether $\omega_{0}$ is rational or not. The right way to do, in order to have a generic family, is to consider $\omega_{0}=\frac{r}{m}(m \geqq 3)$ and play with a "detuning" parameter to allow the argument of the perturbed eigenvalue $\lambda$ (close to $\lambda_{0}$ ) to vary in a neighborhood of $2 \pi \omega_{0}$. The idea is to compute the normal form in the worst case $\left(\omega_{0}\right.$ rational $)$ to have smooth dependency of the coefficients in the parameters. Hence, by essence we need here 3 parameters (defined below) to unfold the singularity. Using the characterization (5) it is easy to show that the normal form is given by:

$$
\left.\begin{array}{l}
x^{\prime}=x+\varphi\left(\mu, x,|z|^{2}, z^{m}, \bar{z}^{m}\right)+O\left[(\|X\|+|\mu|)^{P}\right] \\
z^{\prime}=\lambda_{0} z+z \cdot \psi_{0}\left(\mu, x,|z|^{2}, z^{m}\right)+\bar{z}^{m-1} \cdot \psi_{1}\left(\mu, x,|z|^{2}, \bar{z}^{m}\right)+O\left[(\|X\|+|\mu|)^{P}\right],
\end{array}\right\}
$$

where $\varphi, \psi_{0}, \psi_{1}$ are polynomials in their arguments, $\varphi$ being real and: $\varphi(0)=\psi_{0}(0)$ $=\frac{\partial \varphi}{\partial x}(0)=0$. After renaming the parameters, we only need $(\mu, v) \in \mathbb{R} \times \mathbb{C}$ given by the principal part of (7):

$$
\left.\begin{array}{l}
x^{\prime}=x+\mu+a_{0} \cdot x+b_{0} \cdot|z|^{2}+\text { h.o.t.; } \\
z^{\prime}=\lambda_{0}(1+v) \cdot z+a_{1} \cdot x z+b_{1} \cdot z|z|^{2}+c_{1} \cdot z^{m-1}+\text { h.o.t.; }
\end{array}\right\}
$$

$\operatorname{Im}(v)$ is the "detuning parameter" discussed above, and all the coefficients $a_{0}, \ldots, c_{1}$ are $C^{\infty}$ functions of the parameters $(\mu, v)$.

The study of all bifurcations for the family of maps (8) in the case $m>3$ is exactly the same as the one for vector fields derived in ([Gu-Ho], Sect. 7.4). One sees on the truncated normal form at quadratic order that there are many cases (case II and III of [Gu-Ho] Sect. 7.4) where bifurcation of a family of invariant circles to invariant $\mathbb{T}^{2}$-tori occurs. These $\mathbb{T}^{2}$-tori here appear as a Hopf bifurcation of fixed points to invariant circles for the map in polar coordinates when we forget the argument of $z$. In fact, if we take account of the full map (7), it is not possible to prove directly the occurrence of such a bifurcation, but just prove the tori exist outside a little neighborhood of the bifurcation surface (obtained with the normal form) which is cylindrical here (independent of $\operatorname{Im} v$ ) in the parameter space.

\subsection{Hopf-Flip Interaction}

Here we assume $\sigma_{0}=\{-1\} U\left\{\lambda_{0}\right\} U\left\{\bar{\lambda}_{0}\right\}$, where $\lambda_{0}=e^{2 i \pi \omega_{0}}$, all eigenvalues being simple, we write (6) again where the eigenvector $\xi_{0}$ belongs now to -1 . Derivation of the normal form is as in Sect. 2.2. The characterization (5) of the normal form gives a functional equation easy to solve: when $\omega_{0}=\frac{r}{m}$ we have the following map
in $\mathbb{R} \times \mathbb{C}$ :

$$
\left.\begin{array}{rl}
x^{\prime}= & -x+x \varphi_{0}\left(\mu, x^{2},|z|^{2}, z^{m}, \bar{z}^{m}\right)+z^{m / 2} \varphi_{1}\left(\mu, x^{2},|z|^{2}, z^{m}\right) \\
& +\bar{z}^{m / 2} \frac{\varphi_{1}\left(\mu, x^{2},|z|^{2}, z^{m}\right)}{O} O\left[\|X\|^{P}\right] \\
z^{\prime}= & \lambda_{0} z+z \cdot \psi_{0}\left(\mu, x^{2},|z|^{2}, z^{m}\right)+\bar{z}^{m-1} \cdot \psi_{1}\left(\mu, x^{2},|z|^{2}, \bar{z}^{m}\right) \\
& +x \cdot z^{(m / 2)+1} \psi_{2}\left(\mu, x^{2},|z|^{2}, z^{m}\right)+\bar{z}^{(m / 2)-1} \cdot \psi_{3}\left(\mu, x^{2},|z|^{2}, \bar{z}^{m}\right)+O\left[\|X\|^{P}\right]
\end{array}\right\}
$$


where terms in $z^{m / 2}$ disappear when $m$ is odd, and $\varphi_{j}, \psi_{j}$ are polynomials in their arguments, $\varphi_{0}$ being real.

If $m$ even $\geqq 8$ or odd $\geqq 5$, the truncated normal form at cubic order has the same symmetry as the codimension two problem studied in [La-Io] whose phase diagram are related to the problem studied in $[\mathrm{Gu}-\mathrm{Ho}]$ Sect. 7.5.

In this case we recover the circle doubling phenomenon studied in [Los 1] which already leads to complicated results for the full map (9), but only needs two parameters. Now, as in the case considered in Sect. 2.2 there are many cases where bifurcation $\mathbb{T}^{1} \rightarrow \mathbb{T}^{2}$ takes place on the truncated normal form. In fact here there is not only the $\mathbb{T}^{1} \rightarrow \mathbb{T}^{2}$-bifurcation but also, in some parameter space region, a family of invariant couples of circles $\mathbb{T}^{1}$ bifurcating into invariant couples of $\mathbb{T}^{2}$-tori (inside a couple each element is mapped to the other) [Io 84]. Here again, if we take account of the full map (9) we shall need the three real parameters $(\mu, \operatorname{Re} v$, $\operatorname{Im} v$ ) as in Sect. 2.2 to obtain similar results as will be described below in Sects. 4 and 5 , with the same techniques.

\subsection{Hopf-Pitchfork Interaction}

Another interesting case which occurs quite frequently is the one considered in Sect. 2.2 but in a presence of a symmetry $S$ which commutes with $\mathscr{F}_{\mu}(\cdot)$. We assume that $S$ acts trivially on $\zeta_{0}$ but not on $\xi_{0}$. Since the symmetry property propagates on the center manifold [Ruel] and on the normal form [Elp \& al], we have a map (7) such that $\varphi$ is odd in $x$ and $\psi_{0}, \psi_{1}$ are even in $x$. Hence we recover a mapping (nearly) like (9), with no terms in $z^{m / 2}$, and $+x$ instead of $-x$ in $x^{\prime}$. As a result, here again we have $\mathbb{T}^{1} \rightarrow \mathbb{T}^{2}$-bifurcations and also $\mathbb{T}^{1}$-pitchfork bifurcations on the truncated normal form. The proofs for the full map are the same as in case 2.3 (same "bubbles" in the same parameter space).

\section{Hopf-Hopf Interaction for Maps}

\subsection{Normal Form}

We now consider the case when $\sigma_{0}=\left\{\lambda_{0}\right\} U\left\{\bar{\lambda}_{0}\right\} U\left\{\lambda_{1}\right\} U\left\{\bar{\lambda}_{1}\right\}$, where $\lambda_{j}=e^{2 i \pi \omega_{j}}$, $j=0,1$, all eigenvalues being simple, and we write:

$$
X=z_{0} \cdot \zeta_{0}+\bar{z}_{0} \cdot \bar{\zeta}_{0}+z_{1} \cdot \zeta_{1}+\bar{z}_{1} \cdot \bar{\zeta}_{1} \in E_{0}
$$

where $\zeta_{j}$ is the eigenvector of $T_{0}$ belonging to $\lambda_{j}, j=0,1$. This normal form was studied by Jost and Zehnder [ $\mathrm{Jo}-\mathrm{Ze}]$ with a two dimensional parameter space. As indicated in 2.1 the map reduces to a map in $\mathbb{C}^{2}:\left(z_{0}, z_{1}\right) \rightarrow\left(z_{0}^{\prime}, z_{1}^{\prime}\right)$ of the form $(\mu$ is a parameter in $\mathbb{R}^{k}$ not yet precised):

$$
\left.\begin{array}{l}
z_{0}^{\prime}=\lambda_{0} z_{0}+n_{0}\left(\mu, z_{0}, \bar{z}_{0}, z_{1}, \bar{z}_{1}\right)+O\left[\|X\|^{P}\right] ; \\
z_{1}^{\prime}=\lambda_{1} z_{1}+n_{1}\left(\mu, z_{0}, \bar{z}_{0}, z_{1}, \bar{z}_{1}\right)+O\left[\|X\|^{P}\right]
\end{array}\right\}
$$

where $n_{j}(\mu, 0)=0, \frac{\partial n_{j}}{\partial z_{k}}(0)=0, j, k=0,1$, and (5) gives here:

$$
n_{j}\left(\mu, \lambda_{0} z_{0}, \bar{\lambda}_{0} \bar{z}_{0}, \lambda_{1} z_{1}, \bar{\lambda}_{1} \bar{z}_{1}\right)=\lambda_{j} n_{j}\left(\mu, z_{0}, \bar{z}_{0}, z_{1}, \bar{z}_{1}\right), \quad j=0,1,
$$


i.e. the normal form is equivariant under the group $\left(\theta_{0}, \theta_{1}\right) \rightarrow\left(\theta_{0}+\omega_{0}, \theta_{1}+\omega_{1}\right)$ of $\mathbb{T}^{2}$, which is discrete if $\left(\omega_{0}, \omega_{1}\right) \in \mathbb{Q}^{2}$, and is the complete group of rotation of $\mathbb{T}^{2}$ if $\omega_{0}$ and $\omega_{1}$ are both irrational and not rationally related. The monomials $z_{0}^{p} z_{0}^{q} z_{1}^{r} \bar{z}_{1}^{s}$, of $n_{0}$ and $n_{1}$ which are not identically 0 satisfy:

$$
\begin{array}{ll}
\omega_{0}(p-q-1)+\omega_{1}(r-s) \in \mathbb{Z} & \text { for } n_{0}, \\
\omega_{0}(p-q)+\omega_{1}(r-s-1) \in \mathbb{Z} & \text { for } n_{1} .
\end{array}
$$

It is natural, as explained in 2.2, to take $\omega_{0}$ and $\omega_{1}$ both rational, paying this fact by the occurrence of at most two "detuning parameters" for the two perturbed eigenvalues near $\lambda_{0}$ and $\lambda_{1}$. As a consequence we need 4 real parameters to derive the normal form in its full unfolding: 2 parameters represent the moduli and 2 others the arguments of the perturbed eigenvalues. For the following study we do not need the global writting of the normal form, it is sufficient to assume:

$$
m_{0} \omega_{0}+m_{1} \omega_{1} \in \mathbb{Z} \text {, has no solution for }\left|m_{0}\right|+\left|m_{1}\right| \leqq N,
$$

then the truncated normal form $P_{\mu}$ of $F_{\mu}$, at order $N-2$ reads:

$$
\left.\begin{array}{l}
z_{0}^{\prime}=\lambda_{0} z_{0}\left[1+\mu_{0}+\psi_{0}\left(\mu,\left|z_{0}\right|^{2},\left|z_{1}\right|^{2}\right)\right] ; \\
z_{1}^{\prime}=\lambda_{1} z_{1}\left[1+\mu_{1}+\psi_{1}\left(\mu,\left|z_{0}\right|^{2},\left|z_{1}\right|^{2}\right)\right] ; \mu=\left(\mu_{0}, \mu_{1}\right) \in \mathbb{C}^{2} ;
\end{array}\right\}
$$

where $\psi_{0}$ and $\psi_{1}$ are polynomials in their two last arguments, depending smoothly on $\mu$, each of them have valuation larger than one and degree $\left[\frac{N-3}{2}\right]$ in
$\left(\left|z_{0}\right|^{2},\left|z_{1}\right|^{2}\right)$. The integer $N$ will be fixed latter.

\subsection{Primary Bifurcation. Normal Hyperbolicity Region}

Since we are only interested in the $\mathbb{T}^{1} \rightarrow \mathbb{T}^{2}$-bifurcation we write the normal form (15) in an easier form for computation. The writing (15) is symmetric in $z_{0}$ and $z_{1}$, so there are two equivalent Hopf bifurcations, the first one stands in the $z_{0}=0$ plane and the second in the $z_{1}=0$ plane; we choose this last one as the primary bifurcation, the other choice leading to equivalent results.

We can now define the primary bifurcating invariant curve for $P_{\mu}$ which, by the rotational invariance is a circle $\Gamma_{0}$ provided that $N \geqq 5$ in Sect. 3.1. This circle is defined by:

$$
z_{1}=0, \quad z_{0}=\varrho_{0} e^{2 i \pi \theta_{0}}, \theta_{0} \in \mathbb{T}^{1}
$$

where

$$
1=\left|1+\mu_{0}+\psi_{0}\left(\mu, \varrho_{0}^{2}, 0\right)\right| .
$$

Equation (17) is solvable with respect to $\varrho_{0}$, provided that:

$$
a_{r}=\operatorname{Re} \frac{\partial \psi_{0}}{\partial X}(0,0,0) \neq 0, \quad \text { (generic hypothesis), }
$$

denoting by $X$ the second argument of $\psi_{0}$. We then obtain:

$$
\varrho_{0}^{2}=-\frac{\mu_{0 r}}{a_{r}}+O\left(|\mu|^{2}\right), \quad \text { where } \mu_{0 r}=\operatorname{Re}\left(\mu_{0}\right) .
$$


We assume in what follows, that:

$$
a_{r}<0
$$

then $\mu_{0 r}>0$ when $\Gamma_{0}$ exists (supercritical bifurcation).

The restriction of $P_{\mu}$ on $\Gamma_{0}$ is simply a rotation:

$$
\begin{aligned}
& \theta_{0} \rightarrow \theta_{0}^{\prime}=\theta_{0}+\omega_{\mu} ; \quad \text { where } \\
& \omega_{\mu}=\omega_{0}+\frac{1}{2 \pi} \arg \left[1+\mu_{0}+\psi_{0}\left(\mu, \varrho_{0}^{2}, 0\right)\right] \\
& =\omega_{0}+\frac{1}{2 \pi}\left[\mu_{0 i}-\left(a_{i} / a_{r}\right) \mu_{0 r}+O\left(|\mu|^{2}\right)\right] ;
\end{aligned}
$$

and

$$
a_{i}=\operatorname{Im} \frac{\partial \psi_{0}}{\partial X} \cdot(0,0,0), \quad \mu_{0 i}=\operatorname{Im} \mu_{0}
$$

The normal hyperbolicity of $\Gamma_{0}$ under $P_{\mu}$ is easily defined with the following change of variables from $\mathbb{C}^{2}$ to $\mathbb{T}^{1} \times \mathbb{R} \times \mathbb{C}$ :

$$
\left(z_{0}, z_{1}\right) \rightarrow(\theta, x, z), \text { with } \quad z=z_{1}, z_{0}=\varrho_{0}(1+x) e^{2 i \pi \theta}, \quad x \text { close to } 0,
$$

$P_{\mu}$ is now a map in $\mathbb{T}^{1} \times \mathbb{R} \times \mathbb{C}:(\theta, x, z) \rightarrow(\Theta, X, Z)$,

$$
\left.\begin{array}{l}
\Theta=\theta+\omega_{\mu}+R_{0}\left(\mu,|z|^{2}\right)+\varepsilon \cdot R_{1}\left(\mu, x,|z|^{2}\right) \\
X=(1-\varepsilon) x+Q_{0}\left(\mu,|z|^{2}\right)+\varepsilon \cdot Q_{1}\left(\mu, x,|z|^{2}\right) \\
Z=z\left\{\lambda_{1}(1+v) \cdot e^{2 i \pi \sigma}+S_{0}\left(\mu,|z|^{2}\right)+\varepsilon \cdot S_{1}\left(\mu, x,|z|^{2}\right)\right\},
\end{array}\right\}
$$

where we define the parameters $\varepsilon, v, \sigma$ by:

$$
\begin{aligned}
\varepsilon & =-2 \varrho_{0}^{2}\left[\frac{\partial \psi_{0}}{\partial X} \cdot\left(\mu, \varrho_{0}^{2}, 0\right) \cdot e^{-2 i \pi \omega_{\mu}}\right]_{r}=2 \mu_{0 r}+O\left(|\mu|^{2}\right) \\
v & =\left|1+\mu_{1}+\psi_{1}\left(\mu, \varrho_{0}^{2}, 0\right)\right|-1=\mu_{1 r}-\left(b_{r} / a_{r}\right) \mu_{0 r}+O\left(|\mu|^{2}\right) \\
\sigma & =\frac{1}{2 \pi} \arg \left[1+\mu_{1}+\psi_{1}\left(\mu, \varrho_{0}^{2}, 0\right)\right]
\end{aligned}
$$

the map:

$$
\left(\mu_{0}, \mu_{1}\right) \rightarrow\left(\varepsilon, v, \sigma, \omega_{0}-\omega_{\mu}\right)
$$

is clearly a local diffeomorphism and then defines new coordinates in the parameter space. From now on we shall write:

$$
\mu=\left(\varepsilon, v, \sigma, \omega_{0}-\omega_{\mu}\right) .
$$

The terms $R_{i}, Q_{i}, S_{i}, i=0,1$, are polynomials in their last arguments depending smoothly on the parameters, the valuation is one in $\left(x,|z|^{2}\right)$ and two for $Q_{1}$, the degree being $N-3$ in $(|z|, x)$. 
When we consider the full family $F_{\mu}$ we expect to have problems near $\varepsilon=0$ and near $v=0$. In the new variables $(\theta, x, z)$ the map $F_{\mu}$ takes the form:

$$
\left.\begin{array}{l}
\Theta=\theta+\omega_{\mu}+R\left(\mu, x,|z|^{2}\right)+\hat{R}(\mu, \theta, x, z, \bar{z}) \\
X=(1-\varepsilon) x+Q\left(\mu, x,|z|^{2}\right)+\hat{Q}(\mu, \theta, x, z, \bar{z}) \\
Z=z\left\{\lambda_{1}(1+v) \cdot e^{2 i \pi \sigma}+S\left(\mu, x,|z|^{2}\right)\right\}+\hat{S}(\mu, \theta, x, z, \bar{z}),
\end{array}\right\}
$$

where $R=R_{0}+\varepsilon \cdot R_{1}$, and similarly for $Q, S$, as defined by (26), and:

$$
\varepsilon^{1 / 2}\{|\hat{R}|+|\hat{Q}|\}+|\widehat{S}|=O\left(\varepsilon^{(N-1) / 2}+|z|^{N-1}\right),
$$

where we used the estimate: $(r+s)^{N-1} \leqq C\left(r^{N-1}+s^{N-1}\right), C>0$. In (28) all functions are smooth in their variables, and estimate (29) says at what order the dependency in $\theta$ (or the breaking of the rotational invariance) arrives.

To prove the persistence of a normal hyperbolic invariant closed curve near $\Gamma_{0}$ under the map $F_{\mu}$, we can use the Ruelle-Takens technique [Ru-Ta] (see also [Lan]). By making the change of variables:

$$
x=(\varepsilon)^{\alpha} \hat{x}, \quad z=(\varepsilon)^{(1 / 2)+\beta} \hat{z}, \quad \alpha, \beta>0,
$$

we obtain a new form of the map where it is easy to see that the condition:

$$
\varepsilon^{N / 4}=o(v),
$$

gives us the right size for the perturbations of the hyperbolic linear part of (28), since the perturbations have to be of smaller size than the rate of normal hyperbolicity of $\Gamma_{0}$, here $\min (\varepsilon, v)$. This result (31) follows from the identity:

$$
\max _{(\alpha, \beta)} \min \left(2 \beta-\alpha, \alpha, \frac{N-4}{2}-\alpha, \frac{N-4}{2}-\beta\right)=\frac{N}{4}-1\left(\text { take } \alpha=\beta=\frac{N}{4}-1\right) .
$$

Figure 2 shows the region of the parameter plane $(\varepsilon, v)$ corresponding to $(31)$.

In Sect. 4 we study what happens in the region $\mathscr{V}$ of Fig. 2 not covered by estimate (31). We shall see that we need the parameter $\sigma$ to be able to obtain a persisting $\mathbb{T}^{1}$.

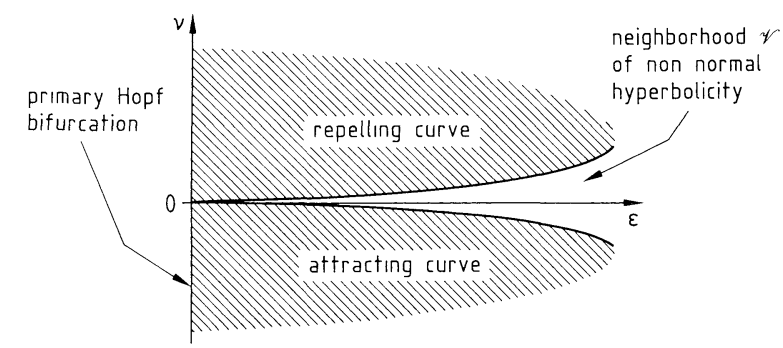

Fig. 2. Persistence of invariant $\mathbb{T}^{1}$ through normal hyperbolicity, $N \geqq 5$ 


\subsection{Secondary Bifurcation. Normal Hyperbolicity Region}

On the truncated normal form $P_{\mu}$ the secondary branch is obtained by looking for $\left(\varrho_{0}, \varrho_{1}\right)$ solution of the system:

$$
\left.\begin{array}{l}
\left|1+\mu_{0}+\psi_{0}\left(\mu, \varrho_{0}^{2}, \varrho_{1}^{2}\right)\right|=1 \\
\left|1+\mu_{1}+\psi_{1}\left(\mu, \varrho_{0}^{2}, \varrho_{1}^{2}\right)\right|=1
\end{array}\right\}
$$

where $\psi_{0}$ and $\psi_{1}$ are defined by (15) and

$$
z_{0}=\varrho_{0} e^{2 i \pi \theta_{0}}, \quad z_{1}=\varrho_{1} e^{2 i \pi \theta_{1}}, \quad\left(\theta_{0}, \theta_{1}\right) \in \mathbb{T}^{2} .
$$

Here we are only interested in what happens near the bifurcation point when $v$ changes its sign. The solution of (32) can be written as follows:

$$
\left.\begin{array}{l}
\varrho_{0}^{2}=-\frac{\varepsilon}{2 a_{r}}+\frac{c_{r} v}{a_{r} d_{r}-b_{r} c_{r}}+O\left(|\mu|^{2}\right) \\
\varrho_{1}^{2}=-\frac{a_{r} v}{a_{r} d_{r}-b_{r} c_{r}}[1+O(|\mu|)],
\end{array}\right\}
$$

where we defined:

$$
c_{r}=\operatorname{Re} \frac{\partial \psi_{0}}{\partial Y}(0,0,0), \quad d_{r}=\operatorname{Re} \frac{\partial \psi_{1}}{\partial Y}(0,0,0),
$$

and where we assume

$$
a_{r} \neq 0, a_{r} d_{r}-b_{r} c_{r} \neq 0,
$$

$Y$ being the last argument of $\psi_{0}$ and $\psi_{1}$. Conditions (34) are generic. Let us now consider the region of the $(\varepsilon, v)$ plane where:

$$
v=o(\varepsilon)
$$

and define $x$ and $y$ by:

$$
\left.\begin{array}{l}
z_{0}=\varrho_{0} \cdot\left(1+x \cdot \varepsilon^{\alpha}\right) e^{2 i \pi \theta_{0}} \\
z_{1}=\varrho_{1} \cdot\left(1+y \cdot \varepsilon^{\alpha}\right) e^{2 i \pi \theta_{1}}
\end{array}\right\}
$$

then the map $F_{\mu}$ written in $\mathbb{R}^{2} \times \mathbb{T}^{2}$ as $\left(x, y, \theta_{0}, \theta_{1}\right) \rightarrow\left(X, Y, \Theta_{0}, \Theta_{1}\right)$ satisfies:

$$
\begin{aligned}
& X=x+2 a_{r} \varrho_{0}^{2}(1+O(|\mu|)) x+2 c_{r} \varrho_{1}^{2}(1+O(|\mu|)) y+O_{1} \\
& Y=y+2 b_{r} \varrho_{0}^{2}(1+O(|\mu|)) x+2 d_{r} \varrho_{1}^{2}(1+O(|\mu|)) y+O_{2} \\
& \Theta_{0}=\theta_{0}+\omega_{0}(\mu)+O_{3} \\
& \Theta_{1}=\theta_{1}+\omega_{1}(\mu)+O_{4}
\end{aligned}
$$

where $\omega_{j}(\mu)$ is defined by

and

$$
\omega_{j}(\mu)=\omega_{j}+\frac{1}{2 \pi} \arg \left[1+\mu_{j}+\psi_{j}\left(\mu, \varrho_{0}^{2}, \varrho_{1}^{2}\right)\right]
$$

$$
O_{i}=O\left(\varepsilon^{\alpha+1}+\varepsilon^{2}+\varepsilon^{-\alpha+(N-2) / 2}+\frac{\varepsilon^{-\alpha+(N-1) / 2}}{|v|^{1 / 2}}\right), \quad i=1,2,3,4
$$




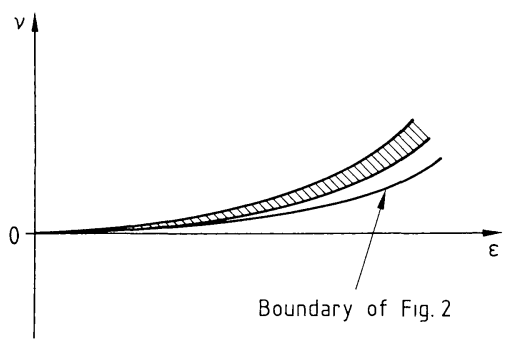

Fig. 3. Persistence of bifurcating invariant $\mathbb{T}^{2}$ through normal hyperbolicity. For the figure we assumed $a_{r} d_{r}-b_{r} c_{r}>0$, hence $v>0$ by (33)

Since the eigenvalues of the matrix:

$$
\left(\begin{array}{ll}
2 a_{r} \varrho_{0}^{2} & 2 c_{r} \varrho_{1}^{2} \\
2 b_{r} \varrho_{0}^{2} & 2 d_{r} \varrho_{1}^{2}
\end{array}\right)
$$

are $-\varepsilon+o(\varepsilon)$ and $-2 v+o(v)$, the normal hyperbolicity argument will give persisting invariant tori, provided that:

$$
\varepsilon^{\alpha+1}+\varepsilon^{2}+\varepsilon^{-\alpha+(N-2) / 2}+\frac{\varepsilon^{-\alpha+(N-1) / 2}}{|v|^{1 / 2}}=o(v) .
$$

This condition is compatible with (35) since we can find: $\alpha=\min \left(2, \frac{N-4}{5}\right)$, and the corresponding region where (35) and (39) hold is indicated at Fig. 3 (for $N=5$ we have $\left.\varepsilon^{6 / 5}=o(v)\right)$.

In Sect. 5 we study what happens for these $\mathbb{T}^{2}$-tori in the region close to the $\varepsilon$-axis, touching the shaded region of Fig. 3 . We shall see that for this bifurcation we need an additional parameter (in fact $\sigma$ ).

\subsection{Remark on Tertiary Bifurcation}

We do not study here the eventual tertiary bifurcation $\mathbb{T}^{2} \rightarrow \mathbb{T}^{3}$, which might be computed again on the truncated normal form, provided that $N \geqq 7$ in (15), if the matrix (38) undergoes a Hopf bifurcation in the $(\varepsilon, v)$ plane. The idea is exactly the same as here, except that there is a degeneracy at cubic order which gives a few more technicalities for the estimates of the normal hyperbolicity region (see the same kind of computations in [La-Io]). For the study inside the non-sufficiently normal hyperbolic region, we need the full unfolding of the family involving the 4 dimensional parameter space defined by (15), to show the persistence of $\mathbb{T}^{2}$-tori as well as for the bifurcating $\mathbb{T}^{3}$-tori. We do not derive this here since this could be done exactly as the case studied in this paper. 


\section{Persistence of an Invariant Curve}

\subsection{First Idea of the Problem}

In this part and the next one we consider, for more clarity, the problem in a 4 dimensional space $E$. This restriction is due to the fact that if we consider the most general family restricted to the center manifold (of dimension 4) this would not allow us to consider a $C^{\infty}$ mapping (see for instance [Ma-Cr], [Io 79]). In Sect. 6 we give a mean to avoid such an assumption. Not making this restriction here should introduce too many inessential complications.

Here we prove the existence of $C^{\infty}$ invariant curves when the parameters lie on some paths going through the region $\mathscr{V}$ where the normal hyperbolicity fails. This family of paths is parametrized by a set which has a Cantor $\times$ Cantor structure, each of these Cantor sets having a positive density of measure in $\mathbb{R}$, near the origin. To prove the persistence of invariant curves Braaksma and Broer [Bra-B] and Broer et al. [BHT], used the Zehnder formulation [Ze] of the strong implicit function theorem. We don't know whether here this would give the full Cantor structure described at the end of this chapter (see the invariant curve theorem).

The method for our proof is similar to the ones in [Chen], [Los 1,2] and is in the spirit of Moser's and Rüssmann's approaches [Mos], [Russ], i.e. we introduce some parameters leading to a "moved" curve theorem, using Herman's formulation [Her], [Bost]. In this case we need to introduce two new parameters, the first one is a rotation parameter as introduced in [Los 1] and the second one corresponds to the "twisted" quasi-conjugacy result obtained in the appendix (Corollary 5 of Theorem A2).

Once this result is obtained we prove that the family of paths described above is given by cancelling these two parameters.

\subsection{Position of the Problem}

We consider the generic family of $C^{\infty}$ diffeomorphisms $(28)$ of $\mathbb{T}^{1} \times(\mathbb{R}, 0) \times(\mathbb{C}, 0)$ obtained in Sect. 3.2, after the change of variables $z=\varepsilon^{1 / 2} z^{\prime}$ and suppressing the primes:

$$
\left.\begin{array}{l}
F_{\mu}(\theta, x, z)=(\Theta, X, Z) ; \mu \in\left(\mathbb{R}^{4}, 0\right) ; \\
\Theta=\theta+\omega_{\mu}+\varepsilon A_{\mu}^{1}\left(x,|z|^{2}\right)+Q_{\mu}^{1}(\theta, x, z, \bar{z}) ; \\
X=(1-\varepsilon) x+\varepsilon A_{\mu}^{2}\left(x,|z|^{2}\right)+Q_{\mu}^{2}(\theta, x, z, \bar{z}) ; \\
Z=e^{2 i \pi\left(\omega_{1}+\sigma\right)}(1+v) z+\varepsilon z A_{\mu}^{3}\left(x,|z|^{2}\right)+Q_{\mu}^{3}(\theta, x, z, \bar{z}) ;
\end{array}\right\}
$$

where $A_{\mu}^{i}, i=1,2,3$ are polynomials of degree $N-3$ in $(x,|z|)$ and of valuation 1 in $\left(x,|z|^{2}\right)$ while $A_{\mu}^{2}$ has valuation 2 , with coefficients depending smoothly on the parameter $\mu \in\left(\mathbb{R}^{4}, 0\right)$. The $Q_{\mu}^{i}, i=1,2,3$ are $C^{\infty}$ perturbations of order $O\left(\varepsilon^{(N / 2)-1}\right)$, where $C^{\infty}$ stands for the parameter and phase space dependencies.

The truncated normal form $N_{\mu}$ defined by $Q_{\mu}^{i} \equiv 0, i=1,2,3$ has a trivial invariant circle:

$$
\mathscr{C}_{0}=\mathbb{T}^{1} \times\{0,0\}
$$


and $N_{\mu \mid \mathscr{C}_{0}}$ is simply a rotation of angle $\omega_{\mu}$. We look for a $C^{\infty}$ invariant closed curve, $C^{1}$-close to $\mathscr{C}_{0}$ for sufficiently small $Q_{\mu}^{i}$. If this curve exists it is described as a graph:

$$
\mathscr{C}=\left\{(\theta, \psi(\theta), \tau(\theta)) ; \theta \in \mathbb{T}^{1}\right\},
$$

where $\psi, \tau$ are $C^{\infty}$ functions on a neighborhood of the origin in $\mathbb{R}$ and $\mathbb{C}$. The invariance of $\mathscr{C}$ under $F_{\mu}$ is made explicit by the following functional equation:

$$
F_{\mu}(\theta, \psi(\theta), \tau(\theta))=\{\Theta(\theta, \psi(\theta), \tau(\theta)), \psi(\Theta), \tau(\Theta)\} .
$$

This kind of equations cannot be solved directly in the non normal hyperbolicity region $\mathscr{V}$ of the parameter space (see Fig. 1). We need, as in the KAM theorem, some assumptions on the rotation properties. Let us first precise some notations which will be used in what follows:

i) $\mathscr{W}_{\mathbb{R}}, \mathscr{W}_{\mathbb{C}}$ are neighborhoods of the origin, respectively in $\mathbb{R}$ and $\mathbb{C} . \mathscr{W}$ is a neighborhood of $\mathbb{T}^{1} \times\{0,0\}$ in $\mathbb{T}^{1} \times \mathbb{R} \times \mathbb{C}$. The study of the family $F_{\mu}$ is now restricted to these neighborhoods.

ii) We introduce the spaces:

$$
\begin{aligned}
& \mathscr{E}=C^{\infty}\left(\mathbb{T}^{1}, \mathscr{W}_{\mathbb{R}}\right) \times C^{\infty}\left(\mathbb{T}^{1}, \mathscr{W}_{\mathbb{C}}\right), \\
& \mathscr{G}=C^{\infty}\left(\mathscr{W}, \mathbb{T}^{1}\right) \times C^{\infty}\left(\mathscr{W}, \mathscr{W}_{\mathbb{R}}\right) \times C^{\infty}\left(\mathscr{W}, \mathscr{W}_{\mathbb{C}}\right),
\end{aligned}
$$

where $C^{\infty}(X, Y)$ is the space of $C^{\infty}$ maps from $X$ to $Y$ both being compact manifolds. By [Ham] these spaces are tame Fréchet spaces for the $C^{\infty}$ topology defined by the countable family of $C^{k}$ uniform convergence norms.

iii) $R_{\omega}$ is the rotation by $\omega$ in $\mathbb{T}^{1}: \theta \rightarrow \theta+\omega$.

iv) Diff ${ }_{+}^{\infty}\left(\mathbb{T}^{1}\right)$ is the group of $C^{\infty}$ diffeomorphisms of the circle preserving the orientation.

\subsection{The Rotated-Twisted Theorem $(\mathrm{R}-\mathrm{T})$}

In this part we prove the key result of the paper in the sense that we define the rotation and the twist parameters which will be cancelled later. Let us define the map:

$$
\left.\begin{array}{l}
\mathscr{R}_{\lambda ; t}(\theta, x, z)=\left(\theta+\lambda, x, e^{2 i \pi t} \cdot z\right) ;(\theta, x, z) \in \mathbb{T}^{1} \times \mathbb{R} \times \mathbb{C} \\
(\lambda ; t) \in \mathbb{T}^{2} .
\end{array}\right\}
$$

For the purpose of this part it is sufficient to consider the following form of the family $F_{\mu}$ :

$$
\left.\begin{array}{l}
F_{\mu}(\theta, x, z)=(\Theta, X, Z) ; \mu \in\left(\mathbb{R}^{4}, 0\right) ; \\
\Theta=\theta+\omega_{\mu}+P^{1}(\theta, x, z, \bar{z}) ; \\
X=(1-\varepsilon) x+P^{2}(\theta, x, z, \bar{z}) ; \\
Z=e^{2 i \pi\left(\omega_{1}+\sigma\right)}(1+v) z+P^{3}(\theta, x, z, \bar{z}) ;
\end{array}\right\}
$$

where $P^{j}=\varepsilon A_{\mu}^{j}+Q_{\mu}^{j}, j=1,2,3$. We write $\Pi=\left(P^{1}, P^{2}, P^{3}\right) \in \mathscr{G}$ in which we have suppressed, for more clarity, the parameter dependencies, even if it is clear that all quantities in what follows depend on $\mu$. 
R-T Theorem. Let $(\omega, \Omega)$ be in $\mathbb{T}^{2}$ and satisfy the diophantine condition:

$$
\exists C>0, \exists \beta \geqq 0 / \forall \frac{p}{q} \in \mathbb{Q},|r \Omega-q \omega-p| \geqq \frac{C}{|q|^{1+\beta}}, \quad r=0,2 .
$$

If $\Pi \in \mathscr{G}$ is sufficiently small, there exists $(\lambda ; t) \in \mathbb{T}^{2},(\psi, \tau) \in \mathscr{E},(a, b) \in\left(\mathbb{R}^{2}, 0\right)$ and $h \in \operatorname{Diff}_{+}^{\infty}\left(\mathbb{T}^{1}\right)$ such that: the closed curve $\mathscr{C}=\left\{(\theta, \psi(\theta), \tau(\theta)) ; \theta \in \mathbb{T}^{1}\right\} \subset \mathbb{T}^{1} \times \mathbb{R} \times \mathbb{C}$ is invariant under $\mathscr{R}_{\lambda ; t} \circ F_{\mu}$. The first projection $\left(\pi_{1}: \mathbb{T}^{1} \times \mathbb{R} \times \mathbb{C} \rightarrow \mathbb{T}^{1}\right)$ of $\left(\mathscr{R}_{\lambda ; t} \circ F_{\mu}\right)_{\mid \mathscr{C}}$ is $C^{\infty}$ conjugated by $h$ to the rotation $R_{\omega}$. The linear part of $\left(\mathscr{R}_{\lambda ; t} \circ F_{\mu}\right)_{\mid \mathscr{G}}$ on the normal bundle over $\mathscr{C}$ is quasi-conjugated (see appendix) to the constant matrix:

$$
\left(\begin{array}{cc}
1-\varepsilon+a & 0 \\
0 & e^{2 i \pi \Omega}(1+v+b)
\end{array}\right)
$$

Moreover the map:

$$
\Pi \rightarrow(\psi, \tau, \lambda, t, h, a, b):(\mathscr{G}, 0) \rightarrow\left[\mathscr{E} \times \mathbb{T}^{2} \times \operatorname{Diff}_{+}^{\infty}\left(\mathbb{T}^{1}\right) \times \mathbb{R}^{2},(0,0) \times\left(\lambda_{0}, t_{0}\right) \times(\mathrm{Id}) \times(0)\right]
$$

is tame, where:

$$
\left.\begin{array}{c}
\lambda_{0}=\omega-\omega_{\mu} \\
t_{0}=\Omega-\omega_{1}-\sigma
\end{array}\right\}
$$

We remark that condition (46) with $r=2$ implies the same condition with $r=1$ (the constant $C$ being different), i.e.:

$$
\exists C>0, \exists \beta \geqq 0 / \forall \frac{p}{q} \in \mathbb{Q},|\Omega-q \omega-p| \geqq \frac{C}{|q|^{1+\beta}} .
$$

These three conditions $(r=0,1,2)$ are needed for the quasi-conjugacy result obtained in Corollary 5 of the appendix.

\subsection{Proof of the $\mathrm{R}-\mathrm{T}$ Theorem}

We first define the following functional operators:

$$
\left.\begin{array}{l}
\Phi: \mathbb{T}^{1} \times \mathscr{E} \times C^{\infty}\left(\mathscr{W}, \mathbb{T}^{1}\right) \rightarrow C^{\infty}\left(\mathbb{T}^{1}\right), \\
\Phi\left(\lambda,(\psi, \tau), P^{1}\right)(\theta)=\theta+\omega_{\mu}+\lambda+P^{1}(\theta, \psi(\theta), \tau(\theta), \bar{\tau}(\theta)),
\end{array}\right\}
$$

and:

$$
\begin{aligned}
& \mathscr{H}_{t}: \mathscr{E} \times \mathscr{G} \rightarrow \mathscr{E} \\
& \left.\mathscr{H}_{t}((\psi, \tau), \Pi)=\left(\begin{array}{l}
\psi \circ \Phi-(1-\varepsilon) \psi-P^{2}(\cdot, \psi, \tau, \bar{\tau}) \\
\tau \circ \Phi-e^{2 i \pi\left(\omega_{1}+\sigma+t\right)}(1+v) \tau-P^{3}(\cdot, \psi, \tau, \bar{\tau})
\end{array}\right) \cdot\right\}
\end{aligned}
$$

To prove the theorem we have to find $((\lambda ; t),(\psi, \tau), h)$ in $\mathbb{T}^{2} \times \mathscr{E} \times \operatorname{Diff}_{+}^{\infty}\left(\mathbb{T}^{1}\right)$ so that:

$$
\begin{gathered}
\Phi\left(\lambda,(\psi, \tau), P^{1}\right)=h^{-1} \circ R_{\omega} \circ h, \\
\mathscr{H}_{t}((\psi, \tau), \Pi)=0 .
\end{gathered}
$$


For the truncated normal form, which is reduced here to the linear part, i.e. $\Pi \equiv 0$ we have trivially:

$$
\left.\begin{array}{l}
\Phi\left(\lambda_{0},(0,0), 0\right)=R_{\omega} \\
\mathscr{H}_{t}((0,0), 0)=0, \text { for any } t .
\end{array}\right\}
$$

The proof of the $\mathrm{R}-\mathrm{T}$ theorem is based on the implicit function theorem on Fréchet spaces in the formulation of Hamilton [Ham]. There are two main steps:

- The conjugacy to the rotation $R_{\omega}$;

- The local inversion of the partial derivative $\frac{\partial \mathscr{H}_{t}}{\partial(t, \psi, \tau)}$ which needs the twisted quasi-conjugacy to a diagonal constant matrix whose complex eigenvalues have arguments $\pm 2 \pi \Omega$ (see the appendix, Corollary 5).

4.4.1. The Conjugacy to the Rotation $R_{\omega}$. The first part of the proof only concerns the map $\Phi$ :

Proposition 1. Let $\omega$ be a diophantine number (satisfying (46) with $r=0$ ). There exists a neighborhood $\mathscr{N}$ of 0 in $\mathscr{E} \times C^{\infty}\left(\mathscr{W}, \mathbb{T}^{1}\right)$ such that for every $\left((\psi, \tau), P^{1}\right)$ in $\mathscr{N}$ there exists: $\lambda_{\omega} \in \mathbb{T}^{1}$ and $h_{\omega} \in \operatorname{Diff}_{+}^{\infty}\left(\mathbb{T}^{1}\right)$ satisfying:

$$
\Phi\left(\lambda_{\omega},(\psi, \tau), P^{1}\right)=h_{\omega}^{-1} \circ R_{\omega} \circ h_{\omega},
$$

the map: $\mathscr{E} \times C^{\infty}\left(\mathscr{W}, \mathbb{T}^{1}\right) \ni\left((\psi, \tau), P^{1}\right) \rightarrow\left(\lambda_{\omega}, h_{\omega}\right) \in \mathbb{T}^{1} \times \operatorname{Diff}_{+}^{\infty}\left(\mathbb{T}^{1}\right)$ is $C^{1}$ tame.

This proposition is a direct consequence of a result by Herman (see [Her 79], Sect. III.4.2) and the Herman-Yoccoz theorem [Yoc]; the $C^{1}$ nature of the map is explicitly proved in [Los 1$]$.

4.4.2. Partial Derivative of the Map $\mathscr{H}$. We study now the map $\mathscr{H}$ where $\lambda$ is given by Proposition 1. In order to apply the theorem of Hamilton to this map we have to prove that the Gâteaux partial derivative:

$$
D_{1} \mathscr{H}_{t} \equiv \frac{\partial \mathscr{H}_{t}}{\partial(t, \psi, \tau)}, \quad \text { admits a local tame inverse }
$$

This means that for every $K$ in $\mathscr{E}$ and every $((\psi, \tau), \Pi)$ in some neighborhood of 0 in $\mathscr{E} \times \mathscr{G}$ the following linear equation:

$$
D_{1} \mathscr{H}_{t}(\psi, \tau, \Pi)(\Delta t, \Delta \psi, \Delta \tau, \Delta \bar{\tau})=K,
$$

has a unique tame solution: $(\Delta t, \Delta \psi, \Delta \tau) \in \mathbb{T}^{1} \times \mathscr{E}$.

By a direct computation we obtain:

$$
\begin{aligned}
D_{1} & \mathscr{H}_{t}(\psi, \tau, \Pi)(\Delta t, \Delta \psi, \Delta \tau, \Delta \bar{\tau}) \\
= & \left(\begin{array}{l}
\Delta \psi \circ \Phi+(D \psi \circ \Phi) \Delta \Phi+A_{11} \Delta \psi+A_{12} \Delta \tau+A_{13} \Delta \bar{\tau} \\
\Delta \tau \circ \Phi+(D \tau \circ \Phi) \Delta \Phi+A_{21} \Delta \psi+A_{22} \Delta \tau+A_{23} \Delta \bar{\tau}+R \Delta t
\end{array}\right),
\end{aligned}
$$

where $\left\{A_{i j}\right\}$ is the Jacobian of the map $(x, z, \bar{z}) \rightarrow(X, Z, \bar{Z})$, calculated at $\left\{(\psi(\theta), \tau(\theta), \bar{\tau}(\theta)), \theta \in \mathbb{T}^{1}\right\}$, for every $(\psi, \tau)$ in some neighborhood of the origin. This matrix is close to the diagonal matrix with eigenvalues:

$$
\left(1-\varepsilon, e^{2 i \pi\left(\omega_{1}+\sigma+t\right)}(1+v), e^{-2 i \pi\left(\omega_{1}+\sigma+t\right)}(1+v)\right) .
$$


By Proposition $1, \lambda(\psi, \tau)$ is a $C^{1}$ tame map, then its derivative:

$\Delta \lambda=\frac{\partial \lambda}{\partial(\psi, \tau)}[\Delta \psi, \Delta \tau, \Delta \bar{\tau}]$ is well defined as a linear functional in $[\Delta \psi, \Delta \tau]$.

By (49) and (54) we have:

$$
\Delta \Phi=\Delta \lambda+\frac{\partial P^{1}}{\partial x}[\cdot, \psi, \tau, \bar{\tau}] \Delta \psi+\frac{\partial P^{1}}{\partial z}[\cdot, \psi, \tau, \bar{\tau}] \Delta \tau+\frac{\partial P^{1}}{\partial \bar{z}}[\cdot, \psi, \tau, \bar{\tau}] \Delta \bar{\tau} .
$$

Gathering (57) and (59) we get:

$$
\begin{aligned}
D_{1} & \mathscr{H}_{t}(\psi, \tau, \Pi)(\Delta t, \Delta \psi, \Delta \tau, \Delta \bar{\tau}) \\
& =\left(\begin{array}{l}
\Delta \psi \circ \Phi+M_{11} \Delta \psi+M_{12} \Delta \tau+M_{13} \Delta \bar{\tau}+L_{1} \Delta \lambda \\
\Delta \tau \circ \Phi+M_{21} \Delta \psi+M_{22} \Delta \tau+M_{23} \Delta \bar{\tau}+R \Delta t+L_{2} \Delta \lambda
\end{array}\right) .
\end{aligned}
$$

The realness of the original problem leads to the following relationships:

$$
\frac{\partial P^{1}}{\partial \bar{z}}=\left(\frac{\overline{\partial P^{1}}}{\partial z}\right), \text { since } P \text { is real, and } \Delta \bar{\tau}=\overline{\Delta \tau} \text {. }
$$

Equation (56) can be written in a vectorial form:

$T \circ \Phi(\theta)+M(\theta) \cdot T(\theta)+L(\theta) \Delta \lambda+R(\theta) \Delta t=K(\theta)$,

where $T=\left(\begin{array}{c}\Delta \psi \\ \Delta \tau\end{array}\right), M=\left\{M_{i j}\right\}, L=\left\{L_{i}\right\}, R=\{0, R, \bar{R}\}, K=\left\{K_{i}\right\}, i, j=1,2,3$,

and $\quad \bar{M}_{12}=M_{13}, \bar{M}_{22}=M_{33}, \bar{M}_{23}=M_{32}, \bar{M}_{21}=M_{31}$.

The complex conjugate of the second component is not written here again, for simplicity.

Let us compose relation (62) with $h^{-1}$ which is given in $\operatorname{Diff}_{+}^{\infty}\left(\mathbb{T}^{1}\right)$ by Proposition 1. We then obtain:

$$
\left.\begin{array}{l}
T^{\prime}(\theta+\omega)=M^{\prime}(\theta) \cdot T^{\prime}(\theta)+L^{\prime}(\theta) \Delta \lambda+R^{\prime}(\theta) \Delta t+K^{\prime}(\theta), \\
\text { where }\left(T^{\prime}, K^{\prime}\right)=(T, K) \circ h^{-1},\left(M^{\prime}, L^{\prime}, R^{\prime}\right)=-(M, L, R) \circ h^{-1},
\end{array}\right\}
$$

and where $\Delta \lambda$ is a linear functional of $T$. By Proposition $1 h$ is in a neighborhood of the identity and is a tame map, then the map $T \rightarrow T^{\prime}$ is a tame isomorphism and the matrix $M^{\prime}$ is in a neighborhood of:

$$
M_{0}=\left(\begin{array}{ccc}
1-\varepsilon & 0 & 0 \\
0 & e^{2 i \pi \Omega}(1+v) & 0 \\
0 & 0 & e^{-2 i \pi \Omega}(1+v)
\end{array}\right)
$$

where $\Omega=\omega_{1}+\sigma+t_{0}$ satisfies condition (46). Then we can apply to the above matrix $M^{\prime}$ the "twisted" quasi-conjugacy result of Corollary 5 of the appendix. 
Thus there exists an $\mathrm{H} \in\left[M_{3}^{\infty}\left(\mathbb{T}^{1}, \mathbb{C}\right)\right.$, id $], \quad D_{r} \in\left[\operatorname{Diag}_{3}(\mathbb{C}), 0\right]$, $D_{\varphi} \in\left[\operatorname{Diag}_{3}(\mathbb{C}), 0\right]$ such that:

$$
\left.\begin{array}{c}
H^{-1}(\theta+\omega)\left[M^{\prime}(\theta)-D_{\varphi}\right] H(\theta)=M_{0}+D_{r}=M_{0}^{\prime}, \text { with, } \\
D_{r}=\left(\begin{array}{ccc}
a & 0 & 0 \\
0 & b e^{2 i \pi \Omega} & 0 \\
0 & 0 & b e^{-2 i \pi \Omega}
\end{array}\right),(a, b) \in\left(\mathbb{R}^{2}, 0\right), \\
D_{\varphi}=\left(\begin{array}{ccc}
0 & 0 & 0 \\
0 & (1+v)\left(1-e^{2 i \pi \varphi}\right) e^{2 i \pi \Omega} & 0 \\
0 & 0 & (1+v)\left(1-e^{-2 i \pi \varphi}\right) e^{-2 i \pi \Omega}
\end{array}\right), \varphi \in(\mathbb{R}, 0) .
\end{array}\right\}
$$

We remark that as $\Omega=\omega_{1}+\sigma+t_{0}$, then the quantity $t_{0}+\varphi$, where $\varphi$ is given by (65) is exactly the parameter $t$ we are looking for. We fix now $t$ to this value which depends functionally on $(\psi, \tau, \Pi)$. The matrix $M^{\prime}(\theta)+D_{\varphi}$ appearing in the quasiconjugacy relation (65) is precisely the new matrix $M^{\prime}$ with the parameter $t$ fixed as above.

The matrix $H$ given by (65) enables us to make the following change of variables (which is tame by Theorem $\mathrm{A} 2$ of the appendix):

$$
T^{\prime}(\theta)=H(\theta) \cdot T^{\prime \prime}(\theta),
$$

which transforms (63) into:

$$
\left.\begin{array}{l}
T^{\prime \prime}(\theta+\omega)=M_{0}^{\prime} \cdot T^{\prime \prime}(\theta)+L^{\prime \prime}(\theta) \Delta \lambda+R^{\prime \prime}(\theta) \Delta t+K^{\prime \prime}(\theta), \\
\text { where } \quad\left(L^{\prime \prime}, R^{\prime \prime}, K^{\prime \prime}\right)(\theta)=H^{-1}(\theta+\omega)\left(M^{\prime}, L^{\prime}, R^{\prime}\right)(\theta) .
\end{array}\right\}
$$

This new equation in $T^{\prime \prime}$ is now solvable, for each $\Delta t, \Delta \lambda$ due to the fact that $M_{0}^{\prime}$ is a constant diagonal matrix whose complex eigenvalue argument satisfies condition (48).

4.4.3. Inversion of the Derivative. Let us be more precise in writing the preceding equation as:

$$
\begin{aligned}
& T^{\prime \prime}(\theta)=\left(\begin{array}{c}
\Delta \psi^{\prime \prime}(\theta) \\
\Delta \tau^{\prime \prime}(\theta) \\
\overline{\Delta \tau^{\prime \prime}(\theta)}
\end{array}\right) \text { with } \Delta \overline{\tau^{\prime \prime}(\theta)}=\overline{\Delta \tau^{\prime \prime}(\theta)} \text {, by }(65) \text { we have: } \\
& \left.\begin{array}{l}
\Delta \psi^{\prime \prime}(\theta+\omega)=(1-\varepsilon+a) \Delta \psi^{\prime \prime}(\theta)+L_{1}^{\prime \prime}(\theta) \Delta \lambda+R_{1}^{\prime \prime}(\theta) \Delta t+K_{1}^{\prime \prime}(\theta), \\
\Delta \tau^{\prime \prime}(\theta+\omega)=(1+v+b) e^{2 i \pi \Omega} \Delta \tau^{\prime \prime}(\theta)+L_{2}^{\prime \prime}(\theta) \Delta \lambda+R_{2}^{\prime \prime}(\theta) \Delta t+K_{2}^{\prime \prime}(\theta),
\end{array}\right\}
\end{aligned}
$$

with $L_{1}^{\prime \prime}, R_{1}^{\prime \prime}, K_{1}^{\prime \prime}$ in $C^{\infty}\left(\mathbb{T}^{1}, \mathbb{R}\right)$ and $L_{2}^{\prime \prime}, R_{2}^{\prime \prime}, K_{2}^{\prime \prime}$ in $C^{\infty}\left(\mathbb{T}^{1}, \mathbb{C}\right)$.

We now want to study the eventual bifurcation $\mathbb{T}^{1} \rightarrow \mathbb{T}^{2}$ in the region $\mathscr{V}$ of the parameter space shown in Fig. 2. In this region the dynamics along the $x$-direction is still normally hyperbolic, while this is not the case in the $z$-plane. In parameter space this can be interpreted by the fact that $v$ can change its $\operatorname{sign}(v=0$ being the locus of the secondary bifurcation for the normal form) while $\varepsilon$ is bounded away from $\varepsilon=0$. 
For our purpose this means that the new hyperbolicity parameter, defined by (65) as $(1-\varepsilon+a)$, is bounded by $(1-\varepsilon+a)<1$, if the perturbation $\Pi$ is small enough in $C^{1}$ topology (a similar result is proved in [Los 2]). This implies that Eq. (68) admits a unique solution in $\Delta \psi^{\prime \prime} \in C^{\infty}\left(\mathbb{T}^{1}, \mathbb{R}\right)$.

For the second Eq. (68) the estimate (48) on $\omega$ and $\Omega$ implies the existence of a solution $\Delta \tau^{\prime \prime} \in C^{\infty}\left(\mathbb{T}^{2}, \mathbb{C}\right.$ ) (see $[\mathrm{Ch}-\mathrm{Io}]$ ), for every $(v, b, \Delta \lambda, \Delta t)$ in $\mathbb{R}^{4}$, the dependency in $\Delta \lambda$ and $\Delta t$ is clearly affine.

We have transformed the initial Eq. (62) into (68) by a finite sequence of tame isomorphisms each of which being close to the identity; solving (68) is then "tamely" equivalent to solve (62), this proves the following:

Proposition 2. Let $(\omega, \Omega)$ satisfy the hypothesis of the $\mathrm{R}-\mathrm{T}$ theorem. Then for every reals $\Delta \lambda, \Delta t$ there exists a tame solution to Eq. (62) which is an affine function in $\Delta \lambda$, $\Delta t$.

The above proposition means that for every reals $\Delta \lambda$ and $\Delta t$ there exist $f_{1}, f_{2}, f_{3}$ in $C^{\infty}\left(\mathbb{T}^{1}, \mathbb{R}\right)$ and $g_{1}, g_{2}, g_{3}$ in $C^{\infty}\left(\mathbb{T}^{1}, \mathbb{C}\right)$ so that:

$$
\left.\begin{array}{l}
\Delta \psi(\theta)=f_{1}(\theta)+f_{2}(\theta) \Delta \lambda+f_{3}(\theta) \Delta t, \\
\Delta \tau(\theta)=g_{1}(\theta)+g_{2}(\theta) \Delta \lambda+g_{3}(\theta) \Delta t,
\end{array}\right\}
$$

is solution of Eq. (62) and then of (56).

We now fix the dependencies of $\Delta \lambda$ and $\Delta t$ in $(\Delta \psi, \Delta \tau)$ which, in turn will prove the existence of a unique $\Delta \psi, \Delta \tau$. We begin by the relation of conjugacy to the rotation $R_{\omega}$ which can be rewritten as:

$$
\Delta \Phi=\Delta \lambda+A_{1} \Delta \psi+A_{2} \Delta \tau+\overline{A_{2}} \cdot \overline{\Delta \tau}=\left[D h^{-1} \circ R_{\omega} \circ h\right]\left(\Delta h-\Delta h \circ h^{-1} \circ R_{\omega} \circ h\right),
$$

composing (70) with $h^{-1}$, which is a tame isomorphism, we obtain:

$$
\left.\begin{array}{l}
\Delta \lambda+A_{1}^{\prime} \Delta \psi^{\prime}+A_{2}^{\prime} \Delta \tau^{\prime}+\overline{A_{2}^{\prime}} \cdot \overline{\Delta \tau^{\prime}}=\left[D h^{-1} \circ R_{\omega}\right]\left(\Delta h^{\prime}-\Delta h^{\prime} \circ R_{\omega}\right), \\
\text { with the notation } X^{\prime}=X \circ h^{-1} \text { for } X=A_{i}, \Delta \psi, \Delta \tau, \Delta h .
\end{array}\right\}
$$

This equation admits a solution if and only if ( $\omega$ satisfying (46)) the following condition is verified:

$$
\int_{\mathbb{T}^{1}} \frac{1}{\left[D h^{-1} \circ R_{\omega}\right]} \cdot\left(\Delta \lambda+A_{1}^{\prime} \Delta \psi^{\prime}+A_{2}^{\prime} \Delta \tau^{\prime}+\overline{A_{2}^{\prime}} \cdot \overline{\Delta \tau^{\prime}}\right) d \theta=0 .
$$

This gives immediately the linear dependency of $\Delta \lambda$ in $(\Delta \psi, \Delta \tau)$. Let us remark that we obtain $\Delta \lambda$ by projecting the relation (71) on the kernel of the commutator $\Delta h^{\prime}-\Delta h^{\prime} \circ R_{\omega}$. This is precisely the method used in the appendix for the quasiconjugacy theorem. By the same method we fix the linear dependency of $\Delta t$ in $(\Delta \psi, \Delta \tau)$. The matrix $M$ considered in Theorem A2 depends, in our case on $(\psi, \tau)$, then the partial derivative (A10) is linear in $(\Delta \psi, \Delta \tau)$. The term $\Delta t$ is the partial derivative:

$$
\Delta t=\frac{\partial t}{\partial(\psi, \tau)}[\Delta \psi, \Delta \tau, \Delta \bar{\tau}]
$$


defined by the matrix $\Delta D_{\varphi}$ (see Sect. A.3.1 of the appendix), which then depends linearly on $(\Delta \psi, \Delta \tau)$ by a relation similar to (72). Finally we have a relation of the form:

$$
\left.\begin{array}{l}
\Delta \lambda=\mathscr{L}_{1}(\Delta \psi, \Delta \tau, \overline{\Delta \tau}), \\
\Delta t=\mathscr{L}_{2}(\Delta \psi, \Delta \tau, \overline{\Delta \tau}),
\end{array}\right\}
$$

where $\mathscr{L}_{i}, i=1,2$ are linear functionals continuous for the $C^{0}$ topology.

Let us observe that the $C^{0}$ norms of $f_{2}, f_{3}, g_{2}, g_{3}$ in (69) are of the same order as the $C^{1}$ norm of $\psi$ and $\tau$ (see (57)), hence they are small. Replacing $\Delta \psi$ and $\Delta \tau$ by their expressions (69) in (73) we obtain a linear inhomogeneous 2 dimensional system in $(\Delta \lambda, \Delta t)$ which is, by the above remark, a small perturbation of an invertible system. In this way we obtain a unique $(\Delta \hat{\lambda}, \Delta t)$, which leads to a unique $(\Delta \psi, \Delta \tau)$ by $(69)$.

This means that the partial derivative $D_{1} \mathscr{H}$ admits a local tame inverse for every $(\psi, \tau, \Pi)$ in some small neighborhood of the origin. This ends the proof of the $\mathrm{R}-\mathrm{T}$ theorem by applying the theorem of Hamilton.

\subsection{Persistence of $C^{\infty}$-Invariant Curves}

To prove the existence of an invariant curve under $F_{\mu}$, it is sufficient to prove that the rotation and the twist parameters may vanish simultaneously. The method for the proof is based on the tame-Lipschitz estimates obtained as consequences of the theorem of Hamilton (see [Ham]). This method has been previously used by Chenciner [Chen] and $\operatorname{Los}[\operatorname{Los} 1,2]$, for similar problems. We only point out here the difficulties and the results since all details of the estimates can be found in the above-mentioned works.

4.5.1. The Genericity Problem. As defined by the $\mathrm{R}-\mathrm{T}$ theorem the parameters $\lambda$ and $t$ depend functionally on $(\psi, \tau) \in \mathscr{E}$. If $(\psi, \tau)$ is fixed by the $\mathrm{R}-\mathrm{T}$ theorem we have in fact two maps $\lambda(\mu)$ and $t(\mu)$. Our goal is to show that equations:

$$
\left\{\begin{array}{l}
\lambda(\mu)=0, \\
t(\mu)=0,
\end{array}\right.
$$

define two local manifolds intersecting transversally, for a given couple $(\omega, \Omega)$ satisfying (46).

Let us recall that $\lambda$ belongs to a neighborhood of $\lambda_{0}=\omega-\omega_{\mu}$ and $t$ to a neighborhood of $t_{0}=\Omega-\omega_{1}-\sigma$. Then $\lambda=0$ and $t=0$ are in these neighborhoods if $\lambda_{0}$ and $t_{0}$ are sufficiently small, which implies:

$$
\left.\begin{array}{l}
\left|\omega-\omega_{0}\right|=O\left(\left|\omega_{0}-\omega_{\mu}\right|\right), \\
\left|\Omega-\omega_{1}\right|=O(|\sigma|) .
\end{array}\right\}
$$

But (74) is not always compatible with the diophantine condition (46) since $\omega_{0}$ is allowed to be rational and $\omega_{1}$ can be in $\omega_{0}$ Q in order to obtain a generic result. Then we need a more restrictive diophantine condition which has been called "with small constant" in [Los 2]: 
Definition 1. The set $D C_{\alpha}\left(\omega_{0}\right)$ of diophantine numbers of "order $\alpha$ " close to $\omega_{0}$ is defined by:

$$
D C_{\alpha}\left(\omega_{0}\right)=\left\{\omega \in \mathbb{T}^{1} / \exists C>0, \exists \beta \geqq 0 / \forall \frac{p}{q} \in \mathbb{Q} / \mathbb{Z},\left|\omega-\frac{p}{q}\right| \geqq \frac{C\left|\omega-\omega_{0}\right|^{\alpha}}{|q|^{2+\beta}}\right\}, \alpha \geqq 0 .
$$

Definition 2. For a fixed $\omega$ in $\mathbb{R}-\mathbb{Q}$ and $\alpha \geqq 0$, we define the set $D C \Omega_{\alpha}\left(\omega_{1}\right)$ of diophantine numbers of "order $\alpha$ " close to $\omega_{1}$ by

$$
D C \Omega_{\alpha}\left(\omega_{1}\right)=\left\{\begin{array}{l}
\Omega \in \mathbb{T}^{1} / \exists C>0, \exists \beta \geqq 0 / \forall \frac{p}{q} \in \mathbb{Q}, \\
|2 \Omega-q \omega-p| \geqq \frac{C\left|\Omega-\omega_{1}\right|^{\alpha}}{|q|^{1+\beta}}
\end{array}\right\} .
$$

The first set has been introduced in [Los 2], it generalizes the standard diophantine set $D C_{0}$ and $D C_{1}$, the Siegel-Moser one [Si-Mo]. The density of measure of $D C_{\alpha}\left(\omega_{0}\right)$ goes to 1 when $\omega$ goes to $\omega_{0}$, for every $\left.\left.\alpha \in\right] 0,1\right]$, and the same holds for $D C \Omega_{\alpha}\left(\omega_{1}\right)$ when $\Omega$ goes to $\omega_{1}$.

Let us now precise the sizes of the neighborhoods defined in the application of the theorem of Hamilton. This is a very technical problem because the new diophantine conditions (75), (76) combined with the estimates (74) introduce some "bad" parameter dependencies for the inverses of operators like $\left(D_{1} \mathscr{H}_{t}\right)^{-1}$. This is due to the fact that each resolution of a difference equation introduces a factor $\frac{1}{\left|\omega-\omega_{0}\right|^{\alpha}}$ or $\frac{1}{\left|\Omega-\omega_{1}\right|^{\alpha}}$ in the estimates of the norms. These estimates only allow us to define exponentially small neighborhoods (with respect to the parameters) on which the theorem of Hamilton is valid (see [Ham], [Chen], [Los 1]). The sizes of these neighborhoods are then much too small for the size $O\left(\varepsilon^{(N / 2)-1}\right)$ of our perturbation. A trick due to Herman allowed Chenciner [Chen] to overcome this difficulty. The general form of the result can be found in ([Los 2], Sect. IV, Lemma 1). In order to apply this result to our case we need the tame estimates of $D_{1} \mathscr{H}_{t}, D_{1}^{2} \mathscr{H}_{t},\left(D_{1} \mathscr{H}_{t}\right)^{-1}$. Let us show the way to obtain the last one, since others are standard.

4.5.2. Estimates of $\left(D_{2} \mathscr{F}\right)^{-1}$ and $\left(D_{1} \mathscr{H}_{t}\right)^{-1}$. The proof of Sect. 4.4 used twice the theorem of Hamilton. The first one was for the quasi-conjugacy result proved in the appendix and the second was for the mapping $\mathscr{H}$. We then need to consider these two problems independently.

A) Estimate for the Quasi-Conjugacy. We assume that $(\omega, \Omega) \in \mathbb{T}^{2}$ which is needed to define the quasi-conjugacy (Corollary 5 of the appendix) satisfies conditions (75), (76). We have noted $\mathscr{F}$ (see A9) the tame map defining the quasi-conjugacy relationship, then we need the tame estimates of $D_{2} \mathscr{\mathscr { F }}, D_{2}^{2} \mathscr{\mathscr { F }},\left(D_{2} \mathscr{F}\right)^{-1}$, in order to apply the result of [Los 2], we only consider the last one since the two others are standard.

The diophantine conditions are used to bound expression $\left(\mathrm{A} 19^{\prime}\right)$ for the proof of Theorem A2. 
Proposition 3. Let $(\omega, \Omega) \in \mathbb{T}^{2}$ satisfy condition (75), (76). The map $\mathscr{F}$ is defined by (A9) and its Gâteaux partial derivative (A11). There exists a positive integer $R>1+\beta$, and for every integer $m$, a positive constant $C_{m}$ (which may depend on $m$ ), such that $\left(D_{2} \mathscr{F}\right)^{-1}$, whose existence is proved in Sect. A.3, satisfies:

$$
\left\|(D \mathscr{F})^{-1}(M, X) W\right\|_{m} \leqq \frac{C_{m}}{\delta}\left\{\|M\|_{m+R}+\|X\|_{m+R}+\|W\|_{m+R}\right\},
$$

where

$$
\delta=\min \left(\left|\omega-\omega_{0}\right|^{\alpha},\left|\Omega-\omega_{1}\right|^{\alpha}\right) .
$$

This kind of estimate is proved in [Chen], [Los 1].

B) The map $\mathscr{H}_{t}$ and the conjugacy to the rotation $R_{\omega}$. We use the same arguments for the map $\mathscr{H}_{t}$ defined by (50) and for the map:

$$
\mathscr{K}\left(\lambda, h, P_{1}\right)(\theta)=\theta+\omega_{\mu}+\lambda+P_{1}(\theta)-h^{-1} \circ R_{\omega} \circ h(\theta),
$$

defining the conjugacy to the rotation $R_{\omega}$. For these two maps we obtain:

Proposition 4. Let $(\omega, \Omega) \in \mathbb{T}^{2}$ satisfy condition (75), (76). The inverses of the partial derivatives $D_{1} \mathscr{H}_{t}$ and $D_{1} \mathscr{K}$ whose existence is proved in Sect. 4.4 satisfy:

$$
\begin{gathered}
\left\|\left(D_{1} \mathscr{H}_{t}\right)^{-1}(\psi, \tau, \Pi) u\right\|_{m} \leqq \frac{C_{m}}{\delta}\left\{\|\psi\|_{m+L}+\|\tau\|_{m+L}+\|\Pi\|_{m+L}+\|u\|_{m+L}\right\} \\
\left\|\left(D_{1} \mathscr{K}\right)^{-1}\left(\lambda, h, P_{1}\right) v\right\|_{m} \leqq \frac{C_{m}}{\delta}\left\{\|h-\operatorname{Id}\|_{m+S}+\|v\|_{m+S}\right\}
\end{gathered}
$$

where $C_{m}$ is a positive constant, L and $S$ are the losses of differentiability, where L and $S>1+\beta$.

4.5.3. Complement to the $\mathrm{R}-\mathrm{T}$ Theorem. The Rotated-Twisted theorem has been proved for the simplified form of $F_{\mu}(45)$ which is sufficient for the existence part of the theorem, but here we want to obtain the estimates for the true family (40). We therefore define precisely the maps $\mathscr{H}_{t}$ and $\mathscr{K}$ in which we only transform, in (50) and (79), the perturbation $\Pi$ by:

$$
\Pi=\varepsilon A_{\mu}+\varepsilon^{(N / 2)-1} \xi_{\mu},
$$

where $\varepsilon^{(N / 2)-1} \xi_{\mu} \in \mathscr{G}$ and $\xi$ is bounded for all $C^{k}$-topologies. The truncated normal form corresponds to $\xi \equiv 0$. As for the quasi-conjugacy map $\mathscr{F}$ in (A9) we define the matrix $M$ by:

$$
M(\theta)=M_{0}+\varepsilon N(\theta),
$$

with $N \in M_{3 C}^{\infty}\left(\mathbb{T}^{1}, \mathbb{R}\right)$, which is also bounded for all $C^{k}$-topologies. In fact by $(57)$ the perturbation matrix $N$ depends on $(\psi, \tau)$ in the following way:

$$
N=O(\psi, \tau)+\varepsilon^{(N / 2)-2} O(1) \text {. }
$$

We precise first the quasi-conjugacy Corollary 5 of the appendix by applying Lemma 1 of [Los 2]. The estimate (77) enables us to define a neighborhood $\mathscr{U}$ of the matrix $M_{0}$ where the quasi-conjugacy relation holds:

$$
\mathscr{U}=\left\{M \in M_{3 C}^{\infty}\left(\mathbb{T}^{1}, \mathbb{R}\right) /\left\|M-M_{0}\right\|_{K} \leqq C \delta^{3}, K \geqq R\right\} .
$$


i) Complement to Corollary 5 of the Appendix. Let us assume that $(\omega, \Omega)$ is in $D C_{\alpha}\left(\omega_{0}\right) \times D C \Omega_{\alpha}\left(\omega_{1}\right)$, then the maps $H, D_{r}, D_{\varphi}$ associated by Corollary 5 with the matrix $M$ in (83) satisfy:

$$
\begin{gathered}
\|H-\mathrm{Id}\|_{m}+\left|D_{r}\right|+\left|D_{\varphi}-D_{t_{0}}\right| \leqq C_{m} \frac{\varepsilon}{\delta}\|N\|_{m+k_{0}} \\
\left\|\frac{\partial H}{\partial \mu}\right\|_{m}+\left|\frac{\partial D_{r}}{\partial \mu}\right|+\left|\frac{\partial D_{\varphi}}{\partial \mu}\right| \leqq C_{m} \frac{\varepsilon}{\delta^{4}}\left\{\|N\|_{m+k_{1}}+\left\|\frac{\partial N}{\partial \mu}\right\|_{m+k_{1}}\right\} .
\end{gathered}
$$

Constants $C_{m}$ are positive and may depend on $m$; the loss of differentiability is represented by $k_{0}$ and $k_{1} \geqq R$.

Remember that, by (65), the parameter $t$ is related to $\varphi$ by: $\varphi=t-t_{0}$. The above result is obtained directly from the estimate (77) and from Lemma 1 of [Los 2]; it contains in particular the estimate we need about the size of the $t_{0}$-neighborhood. We have the same type of result for the maps $\mathscr{H}_{t}$ and $\mathscr{K}$, the goal being to estimate the size of the $\lambda$-neighborhood and also the derivative of $\lambda$ with respect to parameters. Since $\lambda$ depends functionally on $(\psi, \tau)$ we first need to estimate their sizes which are given by the map $\mathscr{H}_{t}$ :

ii) Complement 1 to the $\mathbf{R}-\mathbf{T}$ Theorem. Let $(\omega, \Omega)$ be in $D C_{\alpha}\left(\omega_{0}\right) \times D C \Omega_{\alpha}\left(\omega_{1}\right)$. The map $(\psi, \tau) \in \mathscr{E}$ associated by the $\mathrm{R}-\mathrm{T}$ theorem with the perturbation $\Pi$ in $(82)$ satisfies:

$$
\begin{gathered}
\|\psi\|_{m}+\|\tau\|_{m} \leqq C_{m} \frac{\varepsilon^{(N / 2)-1}}{\delta}\|\xi\|_{m+k_{2}}, \\
\left\|\frac{\partial \psi}{\partial \mu}\right\|_{m}+\left\|\frac{\partial \tau}{\partial \mu}\right\|_{m} \leqq C_{m} \frac{\varepsilon^{(N / 2)-1}}{\delta^{4}}\left\{\|\xi\|_{m+k_{3}}+\left\|\frac{\partial \xi}{\partial \mu}\right\|_{m+k_{3}}\right\} .
\end{gathered}
$$

We obtain the same kind of estimates for the map $\mathscr{K}$.

iii) Complement 2 to the R-T Theorem. With the above hypothesis, the map $\lambda, h$ defined by the $\mathrm{R}-\mathrm{T}$ theorem for the perturbation $\Pi$ given by $(82)$, and $(\psi, \tau)$ satisfying (87), (88) satisfy:

$$
\begin{gathered}
\left|\lambda-\lambda_{0}\right|+\|h-\mathrm{id}\|_{m} \leqq C_{m} \frac{\varepsilon^{(N / 2)-1}}{\delta^{2}}\|\xi\|_{m+k_{4}}, \\
\left|\frac{\partial \hat{\lambda}}{\partial \mu}-\frac{\partial \lambda_{0}}{\partial \mu}\right|+\left\|\frac{\partial h}{\partial \mu}\right\|_{m} \leqq C_{m} \frac{\varepsilon^{(N / 2)-1}}{\delta^{8}}\left\{\|\xi\|_{m+k_{5}}+\left\|\frac{\partial \xi}{\partial \mu}\right\|_{m+k_{5}}\right\} .
\end{gathered}
$$

Gathering (86) and (90) parameters $\delta$ and $\varepsilon$ have to satisfy:

$$
\delta>\max \left(\left(\varepsilon^{(N / 2)-1}\right)^{1 / 8}, \varepsilon^{1 / 4}\right),
$$

in order for the preceding estimates to be bounded when parameters go to 0 . Since $\delta$ is small, this implies that the perturbation order $N$ has to be larger than 4 , which is not new since we already assumed in Sect. 3 that $N \geqq 5$ (minimal value for $N$ to insure normal hyperbolicity outside of $\mathscr{V}$ ). This minimal value is sufficient again by the above estimates; indeed the new hyperbolicity parameter is, by the $\mathrm{R}-\mathrm{T}$ 
theorem: $(1-\varepsilon+a)$, which was assumed in Sect. 4.4 .3 to satisfy $(1-\varepsilon+a)<1$. This implies that $a=o(\varepsilon)$ and, by (83), $\left(83^{\prime}\right),(85),(87),(91)$ this last condition can be satisfied if $N \geqq 5$. Then our method enables us to get the minimal condition on the perturbation order $N-1$.

4.5.4. The Invariant Curve Theorem. Let us recall that: $\delta=\min \left[\left|\omega-\omega_{0}\right|^{\alpha}\right.$, $\left.\left|\Omega-\omega_{1}\right|^{\alpha}\right]$, and by (21), (47), (74) we have:

$$
\delta=O\left[|\sigma|^{\alpha},\left|\mu_{0 i}-\varepsilon / 2 \frac{a_{i}}{a_{r}}\right|^{\alpha}\right] .
$$

In order to avoid unnecessary complications we make the following generic assumption:

$$
a_{i} \neq 0 \text { (where } a_{i} \text { is defined by (22)) . }
$$

This is a twist condition, analogous to the one needed to prove the KAM theorem (see [Her 83]). This condition is not necessary but it shows that the bifurcation we consider is generically of codimension 3 (the parameter $\mu_{0 i}$ being omitted). With assumption $\delta=\varepsilon^{\alpha}$, condition (91) implies:

$$
\alpha<\min [1 / 4,(N / 2-1) / 8] \text {. }
$$

Since we assumed $N \geqq 5$, let us fix $\alpha=1 / 8$, then the $\lambda_{0}$-neighborhood, defined by (89) now reads:

$$
\left|\lambda-\lambda_{0}\right| \leqq C \varepsilon^{5 / 4}
$$

If we choose $\omega$ in a $\omega_{0}$-neighborhood so that

$$
\left|\omega-\omega_{\mu}\right| \leqq K \varepsilon^{5 / 4}, K<C,
$$

then the origin lies in the $\lambda_{0}$-neighborhood (93). Moreover since $a_{i} \neq 0,(90)$ implies that $\frac{\partial \lambda}{\partial \varepsilon} \neq 0$, thus the classical implicit function theorem implies that the equation $\lambda(\mu)=0$ defines a local smooth manifold of the form:

$$
\varepsilon=2 a_{r} \mu_{0 i} / a_{i}+2 \pi 2 a_{r}\left(\omega-\omega_{0}\right) / a_{i}+O\left(\varepsilon^{5 / 4}\right), \text { for } \mu_{0 i}=O(\varepsilon) .
$$

The study of equation $t(\mu)=0$ is performed in a similar way, with the above choices, and by $(83),\left(83^{\prime}\right),(85)$ the $t_{0}$-neighborhood takes the form:

$$
\left|t-t_{0}\right| \leqq C \varepsilon^{5 / 4}
$$

then choosing $\Omega$ in a $\omega_{1}$-neighborhood of the form:

$$
\left|\Omega-\omega_{1}-\sigma\right| \leqq K \varepsilon^{5 / 4}, K<C
$$

insures that the origin lies in the $t_{0}$-neighborhood (96), and by (86) we have: $\frac{\partial t}{\partial \sigma} \neq 0$. This implies the existence of a local smooth manifold on which $t(\mu)=0$; this manifold is clearly transversal to the one defined by (95) and takes the form:

$$
\sigma=\Omega-\omega_{1}+O\left(\varepsilon^{5 / 4}\right)
$$




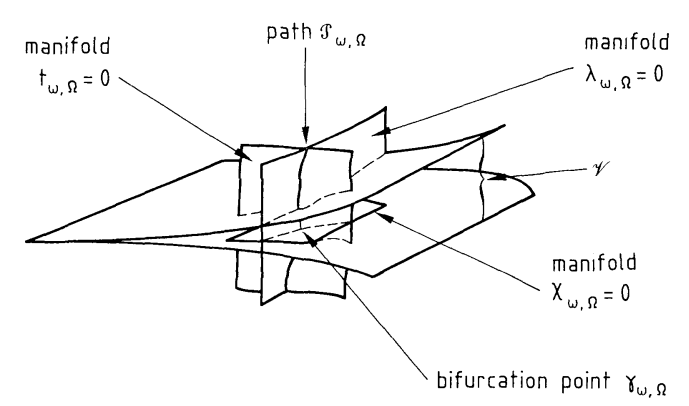

Fig. 4. The transversal manifolds defining the bifurcation point $\gamma_{\omega, \Omega}$

At this point we have proved the main result of the paper, namely there exists a family of paths $\mathscr{P}_{\omega, \Omega}$ in the three dimensional parameter space corresponding to a constant $\mu_{0 i}$ section. Each of these paths is defined by the intersection of the transversal manifolds $\{\mu ; \lambda(\mu)=0\}$ and $\{\mu ; t(\mu)=0\}$ (see Fig. 4) and is such that the one parameter subfamily $\left\{F_{\mu}\right\}_{\mu \in \mathscr{P}_{\omega, \Omega}}$ admits a family of $C^{\infty}$ invariant curves. The set of these paths is parametrized by the set of pairs $(\omega, \Omega)$ satisfying:

$$
\left.\begin{array}{l}
(\omega, \Omega) \in D C_{1 / 8}\left(\omega_{0}\right) \times D C \Omega_{1 / 8}\left(\omega_{1}\right),(\text { see }(75),(76)), \\
\left|\omega-\omega_{\mu}\right| \leqq K \varepsilon^{5 / 4}, \\
\left|\Omega-\omega_{1}-\sigma\right| \leqq K \varepsilon^{5 / 4},
\end{array}\right\}
$$

which is a Cantor set having a positive measure in $\mathbb{T}^{2}$. The above estimates enable us to be more precise about the dynamics, at least for parameter $\mu$ on the paths $\mathscr{P}_{\omega, \Omega}$. Indeed by (85), (86), and from the definition (65) of the matrix $D_{r}$, we define the new parameter:

$$
\chi=v+b,
$$

which characterizes the weak normal hyperbolicity of the invariant curves on $\mathscr{P}_{\omega, \Omega}$. By the above arguments, the equation $\chi(\mu)=0$ defines a smooth local manifold of the form:

$$
v=O\left(\varepsilon^{5 / 4}\right),
$$

which is obviously transversal to the two preceding ones (see Fig. 4).

Now the map $(\varepsilon, v, \sigma) \rightarrow(\lambda, \chi, t)$ defined on a constant $\mu_{0 i}$ section is a local diffeomorphism of $\mathbb{R}^{3}$ containing $(0,0,0)$ in its image, which enables us to define new local parameter space coordinates. The point

$$
\gamma_{\omega, \Omega}=\{(\varepsilon, \nu, \sigma) /(\lambda, \chi, t)=(0,0,0)\},
$$

which belongs to the path $\mathscr{P}_{\omega, \Omega}$ is such that the $C^{\infty}$ invariant curve is non-normally hyperbolic, so it is the bifurcation point corresponding to the pair $(\omega, \Omega)$ in $\mathbb{T}^{2}$. This ends the proof of the following theorem: 
Invariant Curve Theorem. For each $(\omega, \Omega)$ in $\mathbb{T}^{2}$ satisfying (99) there exists a path $\mathscr{P}_{\omega, \Omega}$ in the three dimensional parameter space ( for a fixed $\mu_{0 i}$ ) along which the family $F_{\mu}(24)$, satisfying the generic assumptions (14) with $N \geqq 5,(18),(34)$, and (92), admits a $C^{\infty}$ invariant curve with a rotation number $\omega$. On each of these paths there exists a unique point $\gamma_{\omega, \Omega}$ for which the $C^{\infty}$ invariant curve is non-normally hyperbolic (bifurcation point).

Remark. The largest is $N$, the largest is the Cantor set for $(\omega, \Omega)$ in $\mathbb{T}^{2}$, since we can choose a larger $\alpha$ in (75), (76) bounded by an $N$-dependent constant.

At this point we can now describe the bifurcations of the family $F_{\mu}$, since along each path $\mathscr{P}_{\omega, \Omega}$ we satisfy the conditions of the Chenciner-Iooss paper [Ch-Io].

\section{Bifurcation of Invariant $\mathbb{T}^{2}$ Tori, Bubble Structure}

\subsection{Position of the Problem}

In this chapter we consider the bifurcation problem for mappings in $\mathbb{R}^{4}$ and we define the parameter space regions of persistence for invariant sets $\left(\mathbb{T}^{1}\right.$ and $\mathbb{T}^{2}$ tori). These regions are defined by a normal hyperbolicity argument and they bound the set of bubbles mentioned in the introduction (see Fig. 1).

We use here the consequences of the rotated-twisted theorem (Sect. 4.3) which enables us to localize the study in the phase space near the rotated-twisted curve and near each bifurcation point $\gamma_{\omega, \Omega}$ in the parameter space.

The method is based on some kind of normal form near the "moved curve." It enables us to introduce naturally the parameters defined in the previous chapter and to consider them as a new parametrization of the family. The method is a generalization for a multiparameter family of the approach of Chenciner [Chen] and Los [Los 1] and is a more precise way to express the results of [Ch-Io]. The method of a normal form near an invariant curve (without parameters) was also used by Douady ([Dou] Sect. III).

To be a little bit more precise we consider first a change of variables which localizes the family in a neighborhood of the rotated-twisted curve. Each element of the normal linear bundle over this curve is then an angular dependent matrix for which the quasi-conjugacy theorem (Sect. A.2.3) holds and is thus used to introduce a new change of variables. We then obtain an expression of the family which makes explicitly new parameters $(\lambda, t, \chi)$ of Sect. 4 appear with angular dependent coefficients.

As for the usual normal form technique we can simplify this expression by a sequence of change of variables, replacing angular dependent coefficients of low order by constants or zero, using the diophantine properties of the numbers $\omega, \Omega$.

In fact there are some additional difficulties due to the introduction of a factor 1 $\frac{1}{\varepsilon^{\alpha}}$ at each step of this normalization, because of the type of diophantine condition (99) we use. We then need to estimate carefully all terms to be able to use the standard fixed point technique of Ruelle-Takens [Ru-Ta] at the end. 


\subsection{First Localization Near the Rotated-Twisted Curve}

Let us come back to the family of maps $F_{\mu}$ :

$$
\left.\begin{array}{l}
\Theta=\theta+\omega_{\mu}+\varepsilon A_{\mu}^{1}\left(x,|z|^{2}\right)+Q_{\mu}^{1}(\theta, x, z, \bar{z}), \\
X=(1-\varepsilon) x+\varepsilon A_{\mu}^{2}\left(x,|z|^{2}\right)+Q_{\mu}^{2}(\theta, x, z, \bar{z}), \\
Z=e^{2 i \pi\left(\omega_{1}+\sigma\right)}(1+v) z+\varepsilon z A_{\mu}^{3}\left(x,|z|^{2}\right)+Q_{\mu}^{3}(\theta, x, z, \bar{z}),
\end{array}\right\}
$$

where $Q_{\mu}^{i}=O\left(\varepsilon^{N / 2-1}\right)$.

The $\mathbf{R}-\mathbf{T}$ theorem says that there exists a curve $\mathscr{C}=\left\{(\theta, \psi(\theta), \tau(\theta)), \theta \in \mathbb{T}^{1}\right\}$ which is invariant under $\mathscr{R}_{\lambda, t} \circ F_{\mu}$. This implies the relation:

$$
\left.\begin{array}{l}
F_{\mu}(\theta, \psi(\theta), \tau(\theta))=\mathscr{R}_{\lambda, t}^{-1} \circ(\Phi, \psi(\Phi), \tau(\Phi)), \\
\text { with } \Phi=h^{-1} \circ R_{\omega} \circ h(\theta),
\end{array}\right\}
$$

i.e. we have the equality:

$$
F_{\mu}(\theta, \psi(\theta), \tau(\theta))=\left(\Phi-\lambda, \psi(\Phi), e^{-2 i \pi t} \tau(\Phi)\right),
$$

which is valid for every $\mu$ in a neighborhood of $\gamma_{\omega, \Omega}=\{\mu ; \lambda=0, t=0, \chi=0\}, \chi$ being defined by (100). The size of this neighborhood is given by (93), (96), (101). For $\mu$ in this neighborhood we consider the following change of variables:

$$
\left.\begin{array}{l}
\left(\begin{array}{l}
x \\
z
\end{array}\right)=\left(\begin{array}{l}
\psi \circ h^{-1}\left(\theta^{\prime}\right) \\
\tau \circ h^{-1}\left(\theta^{\prime}\right)
\end{array}\right)+H\left(\theta^{\prime}\right)\left(\begin{array}{l}
x^{\prime} \\
z^{\prime}
\end{array}\right), \\
\theta=h^{-1}\left(\theta^{\prime}\right),
\end{array}\right\}
$$

where $\psi, \tau, h, \lambda, t$, are defined by the $\mathrm{R}-\mathrm{T}$ theorem, and the angular dependent matrix $H$ is given by the quasi-conjugacy theorem (Sect. A.2.2). Let us remark that in a neighborhood of the point $\gamma_{\omega, \Omega}$, the quasi-conjugacy is not exact but is only a "twisted" quasi-conjugacy which introduces a $t$-dependent term in the linear part of the map.

The new form of the mapping is now:

$$
\begin{gathered}
\Theta^{\prime}=\theta^{\prime}+\omega-\lambda+\varepsilon\left|z^{\prime}\right|^{2} f_{1}(\mu)+\varepsilon x^{\prime} f_{2}\left(\mu, \theta^{\prime}, x^{\prime}, z^{\prime}, \bar{z}^{\prime}\right)+\varepsilon^{5 / 4} f_{3}\left(\mu, \lambda, t, \theta^{\prime}, x^{\prime}, z^{\prime}, \bar{z}^{\prime}\right) \\
+O\left(\varepsilon^{3 / 2}\left|z^{\prime}\right|^{4}\right), \\
X^{\prime}=(1-\varepsilon+a) x^{\prime}+\varepsilon\left|z^{\prime}\right|^{2} g_{1}(\mu)+\varepsilon x^{\prime}\left|z^{\prime}\right|^{2} g_{2}\left(\mu, x^{\prime},\left|z^{\prime}\right|^{2}\right)+\varepsilon x^{\prime 2} g_{3}\left(\mu, \theta^{\prime}, x^{\prime}, z^{\prime}, \bar{z}^{\prime}\right) \\
+\varepsilon^{5 / 4} g_{4}\left(\mu, \lambda, t, \theta^{\prime}, x^{\prime}, z^{\prime}, \bar{z}^{\prime}\right)+O\left(\varepsilon^{3 / 2}\left|z^{\prime}\right|^{4}\right), \\
Z^{\prime}=e^{2 i \pi \Omega}\left(1+\chi^{\prime}\right) z^{\prime}+\varepsilon z^{\prime}\left|z^{\prime}\right|^{2} h_{1}(\mu)+\varepsilon^{5 / 4} h_{2}\left(\mu, \lambda, t, \theta^{\prime}, z^{\prime}, \bar{z}^{\prime}\right) \\
\quad+\varepsilon x^{\prime} h_{3}\left(\mu, \lambda, t, \theta^{\prime}, x^{\prime}, z^{\prime}, \bar{z}^{\prime}\right)+O\left(\varepsilon^{3 / 2}\left|z^{\prime}\right|^{4}\right),
\end{gathered}
$$

where

$$
\begin{aligned}
& f_{3}=O\left(|\lambda|+\varepsilon^{1 / 2}\left|z^{\prime}\right|+\left|x^{\prime}\right|\right), g_{4}=O\left\{|\lambda|+|t|+\varepsilon^{1 / 2}\left|z^{\prime}\right|\left(\left|x^{\prime}\right|+\varepsilon^{1 / 2}\left|z^{\prime}\right|\right)\right\}, \\
& h_{2}=O\left(|\lambda|+|t|+\left|z^{\prime}\right|\right), \text { and } \chi^{\prime}=\chi-2 i \pi t(1+v)+O\left(\varepsilon^{5 / 4}+t^{2}\right) .
\end{aligned}
$$

To put this map into normal form we proceed, as in [Ch-Io], by making a sequence of changes of variables close to identity of the form, (suppressing the primes into (105)-(107)):

$$
u^{\prime}=u+\alpha(\theta) \cdot x^{r} \cdot z^{p} \cdot \bar{z}^{q} \cdot \lambda^{s} \cdot t^{n} \text {. with } u=\theta, x, z,
$$


which enables us to simplify the expression of the family $F_{\mu}$ at each step, provided that the following equation admits a solution:

$$
\left.\begin{array}{l}
\alpha(\theta+\omega)(1-\varepsilon)^{m} e^{2 i \pi k \Omega}-\alpha(\theta)=a(\theta), \\
\text { with } m=r(\text { or } r-1 \text { if } u=x) \text { and } k=p-q(\text { or } p-q-1 \text { if } u=z),
\end{array}\right\}
$$

$a(\theta)$ being the angular dependent coefficient ( $C^{\infty}$ function) of the monomial $x^{r} \cdot z^{p} \cdot \bar{z}^{q} \cdot \lambda^{s} \cdot t^{n}$ obtained at each step in the corresponding component of $F_{\mu}$. The strategy consists in beginning with $s=n=0$ and increasing $p+r+q$ up to the desired form, then making $s+n=1$ with the same strategy and so on.

Each time when the exponent of $(1-\varepsilon)$ is not 0 , we can solve Eq. (109) in $\alpha$, provided that the corresponding $a(\theta)$ is of order $\varepsilon$, since the inverse of the left-hand side produces a factor $\varepsilon^{-1}$. We observe that each term containing $x$ in $\Theta$ and $Z$ is at least of order $\varepsilon$. In $X$ we have to look for terms with a power in $x$ distinct of 1 , and here again we see that $\varepsilon$ is in factor. This shows that we can suppress these terms up to the desired order.

For other terms, we have to solve a difference equation like the ones treated in [Ch-Io]. For the case $k=0$, i.e. $p=q$ (respectively $p=q+1$ ) we use the standard diophantine condition on $\omega$ to solve (109) with a right-hand side of 0 mean value. This means that the corresponding coefficients in the expression of the map $F_{\mu}$ will be transformed into a constant (the mean value).

For other values of $(p, q)$ we need non-resonance conditions and diophantine assumptions on $(\omega, \Omega)$ as follows (see (99), (75), (76) for $r=1,2)$ :

$$
\left.\begin{array}{l}
\Omega \neq 0,1 / 2,1 / 3,2 / 3,1 / 4,3 / 4, \\
\exists C>0, \exists \beta \geqq 0 / \forall \frac{p}{q} \in \mathbb{Q},|r \Omega-q \omega-p| \geqq \frac{C \varepsilon^{1 / 8}}{|q|^{1+\beta}}, \\
\text { for } \quad r=0,1,2,3,4(0,3,4 \text { are sufficient }),
\end{array}\right\}
$$

which implies the existence of a solution $\alpha(\theta)$ for Eq. (109), but with a uniform bound on each $C^{k}$ norm multiplied by $\varepsilon^{-1 / 8}$. It can be shown that this does not really give any problem because of the estimates in $\varepsilon^{5 / 4}$ (at least) for every $a(\theta)$ concerned. This shows that our changes of variables can be done up to the stage we wish, corresponding to $p+q \leqq 4$ in $\Theta$ and $X$ and $p+q \leqq 3$ in $Z$ (due to the eventual terms in $\bar{z}^{4}$ in $Z$ ).

We remark that the diophantine conditions (110) are more restrictive than the ones ((75), (76)) needed to prove the invariant curve theorem (Sect. 4.5.4), this is due to the fact that we want to define the paraboloidal regions of normal hyperbolicity with a sufficient size to get through the neighborhood $\mathscr{V}$ (see Figs. 2 and 3 ) and compatible with the optimal value of the perturbation order $(N=5)$. But if we allow a sufficiently large perturbation order the conditions (75), (76) become sufficient (for the persistence analysis).

Finally the mapping takes the form:

$$
\left.\begin{array}{l}
\Theta=\theta+\omega-\hat{\lambda}+\varepsilon|z|^{2}\left[a_{0}(\mu)+a_{1}(\mu, \varepsilon, \lambda, t)\right]+\Theta_{1}, \\
X=(1-\hat{\varepsilon}) x+\varepsilon^{2} x|z|^{2}\left[b_{0}(\mu)+b_{1}(\mu, \varepsilon, \lambda, t)\right]+X_{1}, \\
Z=e^{2 i \pi \Omega}(1+\hat{\chi}) z+\varepsilon z|z|^{2}\left[c_{0}(\mu)+c_{1}(\mu, \varepsilon, \lambda, t)\right]+Z_{1},
\end{array}\right\}
$$


where:

$$
\left.\begin{array}{l}
\hat{\lambda}=\lambda\left(1+O\left(\varepsilon^{5 / 4}\right)\right), \\
\hat{\varepsilon}=\varepsilon+O\left(\varepsilon^{5 / 4}\right), \\
\hat{\chi}_{r}=\chi+O\left(\varepsilon^{5 / 4}\right)+O\left(t^{2}\right), \\
\hat{\chi}_{i}=-2 \pi t\left[1+v+O\left(\varepsilon^{5 / 4}\right)+O(t)\right],
\end{array}\right\}
$$

and we recall that (see Sect. 4):

$$
\left.\begin{array}{l}
\lambda=\omega-\omega_{\mu}+O\left(\varepsilon^{5 / 4}\right), \\
t=\Omega-\omega_{1}-\sigma+O\left(\varepsilon^{5 / 4}\right), \\
\chi=v+O\left(\varepsilon^{5 / 4}\right) .
\end{array}\right\}
$$

Moreover we now have the estimates:

$$
\left.\begin{array}{l}
\Theta_{1}=O\left(\varepsilon|x|^{m_{0}}+\varepsilon^{3 / 2}|z|^{4}+\varepsilon^{5 / 4}|\lambda|^{k_{0}}\right), \\
X_{1}=O\left(\varepsilon|x|^{m_{1}}+\varepsilon^{3 / 2}|z|^{4}+\varepsilon^{5 / 4}|\lambda|^{k_{1}}\right), \\
Z_{1}=O\left(\varepsilon|x|^{m_{2}}+\varepsilon^{3 / 2}|z|^{4}+\varepsilon^{5 / 4}|\lambda|^{k_{2}}\right),
\end{array}\right\}
$$

where we denote by $|\lambda|$ the norm of $(\lambda, t)$, and $a_{1}, b_{1}, c_{1}=O\left(\varepsilon^{1 / 4}\right)$.

\subsection{Persistence of an Invariant $\mathbb{T}^{1}$-Curve}

Let us introduce scales $\xi$ and $\zeta$ as follows:

$$
\left.\begin{array}{l}
x=\xi x^{\prime}, \\
z=\zeta z^{\prime} .
\end{array}\right\}
$$

The idea is to use the graph transform technique, such as used by Ruelle-Takens $[\mathrm{Ru}-\mathrm{Ta}]$ and Lanford [Lan] to prove the existence of an invariant curve $x^{\prime}=\psi^{\prime}(\theta)$, $z^{\prime}=\tau^{\prime}(\theta)$ in a certain domain of the parameter space defined by inequalities on $\lambda, t, \tilde{\chi}$ (where $\tilde{\chi}=|1-\hat{\chi}|-1$ ) and $\varepsilon$.

We start with the assumption that $\tilde{\chi}=o(\varepsilon)$ and in fact we define: $\tilde{\chi}=\bar{\chi} \varepsilon^{\varrho}$, with $\varrho<\frac{6}{5}$ in such a way that when $\bar{\chi}=O(1)$ the corresponding parameter space domain is larger than the neighborhood $\mathscr{V}$ defined in Sects. 3.2-3.3 (see Figs. 2 and 3).

Let us denote by $\Theta_{2}, X_{2}, Z_{2}$, the expressions given by:

$$
\left.\begin{array}{l}
\Theta=\theta+\omega-\hat{\lambda}+\Theta_{2}, \\
X^{\prime}=(1-\hat{\varepsilon}) x^{\prime}+X_{2}, \\
Z^{\prime}=e^{2 i \pi \Omega}(1+\hat{\chi}) z^{\prime}+Z_{2},
\end{array}\right\}
$$

then, to be able to use the graph transform method it is sufficient to arrange things so that:

$$
\left.\begin{array}{l}
\Theta_{2} \text { and its derivatives in } x^{\prime}, z^{\prime} \text { are } o(\tilde{\chi}), \\
X_{2} \text { and its derivatives in } x^{\prime}, z^{\prime} \text { are } o(\varepsilon), \\
Z_{2} \text { and its derivatives in } x^{\prime}, z^{\prime} \text { are } o(\tilde{\chi}),
\end{array}\right\}
$$




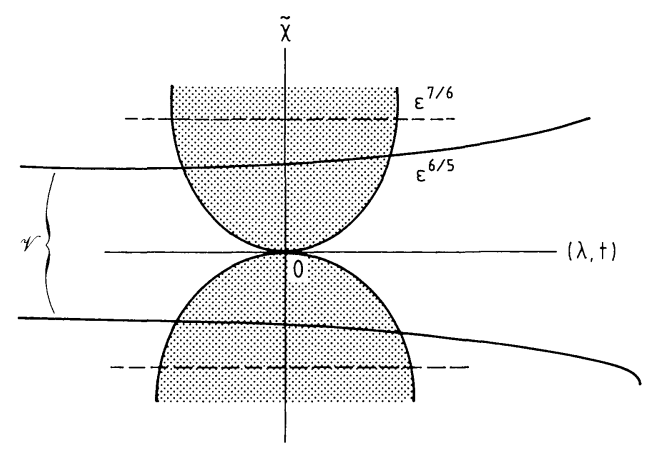

Fig. 5. Region of persistence of an invariant closed curve for $F_{\mu}$, for a fixed $(\omega, \Omega)$. The $\tilde{\chi}$ axis corresponds to a path $\mathscr{P}_{\omega, \Omega}$ found at Sect. 4

It is easy to show that, if we choose $\varrho=\frac{7}{6}, m_{0}=m_{1}=m_{2}=2, k_{0}=k_{1}=k_{2}=1$ and the scales $\zeta=\bar{\chi}^{1 / 2} \varepsilon^{1 / 4}, \xi=\bar{\chi}^{3 / 4} \varepsilon^{1 / 4}$, then for:

$$
|\lambda|=o\left(\bar{\chi}^{3 / 4} \varepsilon^{1 / 8}\right)=o\left(\frac{\tilde{\chi}^{3 / 4}}{\varepsilon}\right),
$$

we can show the persistence of an invariant $\mathbb{T}^{1}$-curve for the map $F_{\mu}$. Let us observe that (117) defines a paraboloidal region (see Fig. 5).

Remark 1. For this persistence result, we can suppress the part of condition (110) with $r=4$ and the non-strong resonance condition for $r=1 / 4,3 / 4$. In chapter 4 we also had not the condition for $r=3$ and $\Omega=1 / 3,2 / 3$, but if we try to escape from this condition, we should have to pay by assuming a larger value than 5 for $N$ in (14), to be able to find a suitable $\varrho<6 / 5$ for $\tilde{\chi}=\bar{\chi} \varepsilon^{\varrho}$.

\subsection{Bifurcation of an Invariant $\mathbb{T}^{2}$-Torus}

Let us follow the same method as in [Ch-Io]. First, we write the map (111) in the polar coordinates:

$$
z=r e^{2 i \pi \theta_{1}}
$$

and we define the mean radius $r_{1}$ of the bifurcating torus by:

$$
r_{1}^{2}=-\frac{\tilde{\chi}}{\varepsilon\left(d_{0}+d_{1}\right)}
$$

where $d_{0}+d_{1}=\operatorname{Re}\left(e^{-2 i \pi \Omega}\left[c_{0}(\mu)+c_{1}(\mu, \varepsilon, \lambda, t)\right](1+\hat{\chi})^{-1}\right)$, is supposed to be strictly negative, to fix the direction of the bifurcating family (the reader can check that $\varepsilon r_{1}^{2}$ is mainly $\varrho_{1}^{2}$ of (33)).

Now we choose scales $\xi$ and $\zeta$ as above:

$$
\left.\begin{array}{l}
x=\xi x^{\prime}, \\
r=r_{1}\left(1+\zeta y^{\prime}\right)
\end{array}\right\}
$$


so that the map $F_{\mu}$ becomes (denoting by $\theta_{0}$ the old $\theta$ ):

$$
\left.\begin{array}{l}
\Theta_{0}=\theta_{0}+\omega-\hat{\lambda}+\varepsilon r_{1}^{2}\left[a_{0}+a_{1}\right]+\Theta_{02}^{\prime}, \\
X^{\prime}=(1-\hat{\varepsilon}) x^{\prime}+X_{2}^{\prime}, \\
Y^{\prime}=(1-2 \tilde{\chi}) y^{\prime}+Y_{2}^{\prime}, \\
\Theta_{1}=\theta_{1}+\Omega+\frac{1}{2 \pi} \arg (1+\hat{\chi})+\varepsilon r_{1}^{2}\left[e_{0}+e_{1}\right]+\Theta_{12}^{\prime},
\end{array}\right\}
$$

where $e_{0}+e_{1}=\frac{1}{2 \pi} \arg \left(e^{-2 i \pi \Omega}\left[c_{0}(\mu)+c_{1}(\mu, \varepsilon, \lambda, t)\right](1+\hat{\chi})^{-1}\right)$, and:

$$
\left.\begin{array}{l}
\Theta_{02}^{\prime}=O\left(\tilde{\chi} \zeta+\varepsilon \xi^{m_{0}}+\frac{\tilde{\chi}^{2}}{\varepsilon^{1 / 2}}+\varepsilon^{5 / 4}|\lambda|^{k_{0}}\right), \\
X_{2}^{\prime}=O\left(\tilde{\chi} \varepsilon+\varepsilon \xi^{m_{1}-1}+\frac{\tilde{\chi}^{2}}{\xi \varepsilon^{1 / 2}}+\varepsilon^{5 / 4} \frac{|\lambda|^{k_{1}}}{\xi}\right), \\
Y_{2}^{\prime}+\Theta_{02}^{\prime}=O\left(\tilde{\chi} \zeta+\frac{\varepsilon^{3 / 2} \xi^{m_{2}}}{\zeta \tilde{\chi}^{1 / 2}}+\frac{\tilde{\chi}^{3 / 2}}{\zeta}+\varepsilon^{7 / 4} \frac{|\lambda|^{k_{2}}}{\zeta \tilde{\chi}^{1 / 2}}\right) .
\end{array}\right\}
$$

By choosing $k_{0}=k_{1}=2, k_{2}=4, m_{0}=1, m_{1}=m_{2}=2$, and:

$$
\tilde{\chi}=\bar{\chi} \varepsilon^{7 / 6} \text { (as for the persistence), } \zeta=\bar{\chi}^{1 / 2} \varepsilon^{1 / 12}, \zeta=\bar{\chi} \varepsilon^{1 / 4},
$$

we can observe that in the following region of the parameter space (see Fig. 6):

$$
|\lambda|^{2}=o\left(\frac{\bar{\chi}}{\varepsilon^{1 / 12}}\right)=o\left(\frac{\tilde{\chi}}{\varepsilon^{5 / 4}}\right),
$$

the existence of a bifurcating family of invariant $\mathbb{T}^{2}$-tori can be proved by the standard graph transform method technique (see [Ru-Ta], [Lan]).

Remark 2. We could use as well a sub-center manifold here to eliminate the variable $x$. The problem is then to take care to the fact that $(1-\hat{\varepsilon})$ is close to 1 , this implying additional difficulties to the usual ones.

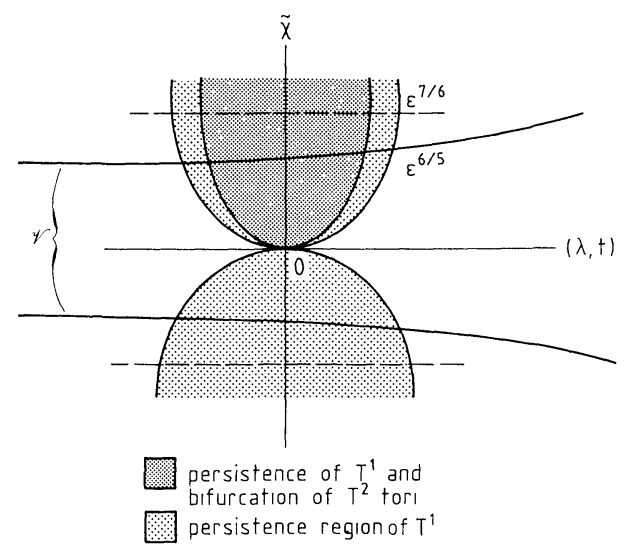

Fig. 6. Regions of persistence of invariant $\mathbb{T}^{1}$ and bifurcating invariant $\mathbb{T}^{2}$ 
Remark 3. We did not try to avoid the diophantine condition (110) for $r=4$, which is probably not necessary, as shown in ([Ch-Io] Sect. V.3), since this would become really very technical for a minor improvement.

Let us sum up the results of this part by the following:

Bubbles-Theorem. For the generic family of diffeomorphisms $F_{\mu}(11)$, satisfying the non-strong resonance condition (14) with $N \geqq 5$ and the non-degeneracy conditions (20), (34), there exists a set of two sided paraboloidal regions tangent at their vertices $\gamma_{\omega, \Omega}$ (see Figs. 5, 6) getting through the neighborhood $\mathscr{V}$ (defined on Sect. 3.3, see Fig. 3). The points $\gamma_{\omega, \Omega}$ are defined, for each $(\omega, \Omega)$ satisfying (110) and (99), by the invariant curve theorem of Sect. 4.

In these regions there is a family of $C^{k}$ invariant $\mathbb{T}^{1}$-curves $\left(C^{\infty}\right.$ on the special path $\mathscr{P}_{\omega, \Omega}$ ) loosing their normal hyperbolicity at each vertex of the paraboloids. Moreover, in each one-sided paraboloidal region (Fig. 6), there bifurcates from each vertex a family of $C^{k}$ normally hyperbolic invariant $\mathbb{T}^{2}$-tori.

Another way to settle this result is to say that in the union of the above paraboloïdal regions the family $F_{\mu}$ "looks like" the truncated normal form $N_{\mu^{\prime}}$ (defined by (24)), for $\mu^{\prime}$ in a neighborhood of $\mu$, in the sense defined by [Chen], [Los 1]. We could formulate this theorem in a very precise setting, but the price to pay is certainly no less than two pages more!

We finally obtained the bubble structure announced in the introduction as the complementary, in the parameter space, of the paraboloidal regions.

\section{Persistence and Bifurcation of Tori in Higher Dimensions}

In this part we generalize in a Banach space $E$ (of dimension higher than 4 !) the results proved, in the preceding Sects. 4 and 5 , for families of mappings in $\mathbb{R}^{4}$. The frame is the one described in Sect. 2.1 with assumptions of Sect. 3. The problem here is that we cannot use the center manifold because we loose the $C^{\infty}$. differentiability in that case.

The key tool is that we can generalize the quasi-conjugacy theorem of the appendix in the infinite dimensional space $E$, in the following sense. Assume that the linear operator function on $\mathbb{T}^{1}: \theta \rightarrow M(\theta)$ is close to a constant linear operator $\mathscr{T}_{0}$ in $E$, with a spectrum separated in two parts $\sigma_{0}$ and $\sigma_{-}$, as described in Sect. 2.1. The idea is that we can uncouple in a $C^{\infty}$ way two vector bundles over $\mathbb{T}^{1}$, invariant under the map: $(\theta, X) \rightarrow(\theta+\omega, M(\theta) X)$, such that on one side ( $\infty$-dimensional) is a strict contraction, while on the other (finite dimensional) we are reduced to the case studied in the appendix. Hence it is only necessary to have the quasiconjugacy to a constant on the finite dimensional part whose spectrograph (in the sense of $[\mathrm{Ch}-\mathrm{Io}])$ is close to the unit circle.

\subsection{Position of the Problem}

Let us consider the linear operator $\mathscr{T}_{0}$ in $\mathscr{L}(E)$ defined in Sect. 2.1, where $\mathscr{L}(E)$ denotes the Banach space of bounded linear operators in $E$. Its spectrum is splitted into two parts $\sigma_{0}$ and $\sigma_{-}$, where $\sigma_{-}$is strictly contained in the open unit disc and $\sigma_{0}$ is a finite set of eigenvalues on the unit circle of finite multiplicities. This 
assumption is relevant for instance for Poincaré maps built with evolution problems such as Navier-Stokes equations [Io 79], where $\mathscr{T}_{0}$ is a compact operator. The space $E$ is then decomposed (see [Kato]) as follows into a sum of invariant subspaces:

$$
E=E_{0} \oplus E_{-}, \operatorname{dim} E_{0}=n,
$$

where the spectrum of the restriction of $\mathscr{T}_{0}$ on $E_{0}$ (respectively $E_{-}$) is $\sigma_{0}$ (respectively $\sigma_{-}$), and we define $T_{0}=\mathscr{T}_{0 \mid E_{0}}, T_{-}=\mathscr{T}_{0 \mid E_{-}}$.

We now consider the $C^{\infty}$ map:

$$
\mathscr{T} \in C^{\infty}\left[\mathbb{T}^{1},\left(\mathscr{L}(E), \mathscr{T}_{0}\right)\right],
$$

and we define the following decomposition for any $Z=X+Y$ in $E$, with $X \in E_{0}$, $Y \in E_{-}$:

$$
\mathscr{T}(\theta) Z=\left[M_{0}(\theta) X+N_{0}(\theta) Y, N_{-}(\theta) X+M_{-}(\theta) Y\right]
$$

where:

$$
\left.\begin{array}{l}
M_{0} \in C^{\infty}\left[\mathbb{T}^{1},\left(\mathscr{L}\left(E_{0}\right), T_{0}\right)\right] \cong\left(M_{n \mathbb{C}}^{\infty}\left(\mathbb{T}^{1}, \mathbb{R}\right), T_{0}\right), \\
M_{-} \in C^{\infty}\left[\mathbb{T}^{1},\left(\mathscr{L}\left(E_{-}\right), T_{-}\right)\right], \\
N_{0} \in C^{\infty}\left[\mathbb{T}^{1},\left(\mathscr{L}\left(E_{-}, E_{0}\right), 0\right)\right], \\
N_{-} \in C^{\infty}\left[\mathbb{T}^{1},\left(\mathscr{L}\left(E_{0}, E_{-}\right), 0\right)\right] .
\end{array}\right\}
$$

Here we denoted by $\mathscr{L}\left(E_{1}, E_{2}\right)$ the Banach space of bounded linear operators from $E_{1}$ to $E_{2}$.

The study made at Sect. 4 shows that the main difficulty for proving the persistence result lies in the linear step of the proof. For the 4 dimensional system the key result is the quasi-conjugacy theorem A.2 of the appendix, by which we can transform the linear part of the map $\mathscr{R}_{\lambda, t} \circ F_{\mu}$, over the $\mathbb{T}^{1}$ torus, into a constant matrix. This kind of result is generally hopeless in infinite dimensions, so we are in fact less ambitious since we just need to uncouple the linear part (127) as follows:

Lemma 6.1. Let $\mathscr{T}$ be given by (127), (128) and be in a sufficiently small neighborhood of $\mathscr{T}_{0}$. Then, for any $\omega$ in $\mathbb{T}^{1}$, there exists a map $\mathscr{H}$ in $C^{\infty}\left[\mathbb{T}^{1},(\mathscr{L}(E), \mathrm{Id})\right]$ depending tamely on $\mathscr{T}$, and such that:

$$
\mathscr{H}^{-1}(\theta+\omega) \cdot \mathscr{T}(\theta) \cdot \mathscr{H}(\theta)=\left(\begin{array}{cc}
M_{0}^{\prime}(\theta) & 0 \\
0 & M_{-}^{\prime}(\theta)
\end{array}\right),
$$

where the matrix representation corresponds to the product space $E_{0} \times E_{-}$and:

i) $\left(M_{0}^{\prime}, M_{-}^{\prime}\right) \in C^{\infty}\left[\mathbb{T}^{1},\left(\mathscr{L}\left(E_{0}\right) \times \mathscr{L}\left(E_{-}\right),\left(T_{0}, T_{-}\right)\right)\right]$ depends tamely on $\mathscr{T}$, and moreover:

ii) $\mathscr{H}(\theta)=\left(\begin{array}{cc}\mathrm{Id} & K(\theta) \\ L(\theta) & \mathrm{Id}\end{array}\right)$, with $L$ (respectively $K$ ) close to 0 in $C^{\infty}\left[\mathbb{T}^{1}, \mathscr{L}\left(E_{0}, E_{-}\right)\right]$(respectively $C^{\infty}\left[\left(\mathbb{T}^{1}, \mathscr{L}\left(E_{-}, E_{0}\right)\right)\right.$ ).

In the purpose of using the theorem of Hamilton [Ham] we now need to verify that the spaces we consider are tame Fréchet spaces [Ham]. This was not necessary in 
Sect. 4 and the appendix since the result is standard for the spaces used there. In fact we have the following:

Lemma 6.2. Let $B$ be a Banach space, then the space $\mathscr{S}^{\infty}=C^{\infty}\left[\mathbb{T}^{1}, B\right]$ is a tame Fréchet space (TFS).

This result can directly be extended to the case when $\mathrm{T}^{1}$ is replaced by a compact manifold. The proof of this result is included here for completeness and is a natural extension of the standard cases.

Proof. With the family of norms:

$$
\|f\|_{n}=\sup _{j \leqq n} \sup _{\theta \in \mathbb{T}^{1}}\left\|D^{j} f(\theta)\right\|, f \in \mathscr{S}^{\infty},
$$

the space $\mathscr{S}^{\infty}$ is a graded Fréchet space, with the grading:

$$
\|\ldots\|_{0} \leqq\|\ldots\|_{1} \leqq \ldots \leqq\|\ldots\|_{n} \leqq \ldots .
$$

Let us recall one possible definition for tame Fréchet spaces:

Definition. A graded Fréchet space $\left\{F,\left(\|\ldots\|_{n}, n \in \mathbb{N}\right)\right\}$ is a tame Fréchet space if there exists a family $\left(S_{t}\right)_{t \in] 1, \infty[}$ of continuous linear maps from $F$ to $F$ such that:

$$
\left.\begin{array}{l}
\exists r \in \mathbb{N} / \forall(k, n) \in \mathbb{N}^{2}, k \leqq n, \exists C_{n, k}>0 \text { such that: } \\
\forall x \in F, \forall t \in] 1, \infty\left[,\left\|S_{t} x\right\|_{n} \leqq C_{n, k} t^{n-k+r}\|x\|_{k},\right. \\
\left\|S_{t} x-x\right\|_{k} \leqq C_{n, k} t^{k-n+r}\|x\|_{n} .
\end{array}\right\}
$$

This definition was the one used by Hamilton in the preprint version [Ham 74] of his theorem (see also [Bost]).

The method for the proof is the same as the one used to prove the equivalent result for the space $C^{\infty}\left[\mathbb{T}^{1}\right]$ (see [Her] Sect. IV.2.5).

Let $\eta \in C^{\infty}[\mathbb{R}]$ such that: $[-1,1] \supset \operatorname{Sup}(\eta), \eta$ even and $\eta(x)=1$ if $|x| \leqq \frac{1}{2}$, and define the following real functions:

$$
\left.\begin{array}{l}
\varphi(x)=\int_{\mathbb{R}} e^{2 i \pi \xi x} \cdot \eta(\xi) d \xi \\
\varphi_{t}(x)=t \varphi(t x) .
\end{array}\right\}
$$

Then we have the following:

Proposition. Let $T$ be in $\mathscr{S}^{0}=C^{0}\left[\mathbb{T}^{1}, B\right]$, then:

$$
S_{t} T=T * \varphi_{t}=\int_{\mathbb{R}} T(\theta-y) \cdot \varphi_{t}(y) d y \quad \text { is in } \mathscr{S}^{\infty} .
$$

$T$ is considered in (134) as a periodic map on the real line. Expanding $T$ in Fourier series, and noticing that we have for any $n \in \mathbb{N}$ :

$$
S_{t}\left(e^{2 i \pi n \theta} \cdot b\right)=\eta\left(\frac{n}{t}\right) \cdot e^{2 i \pi n \theta} \cdot b, \quad \forall b \in B .
$$

The definition of $\eta$ implies that $S_{t} T$ is a trigonometric polynomial of degree $d \leqq|t|$. The family $\left(S_{t}\right)_{t \in] 1, \infty[}$ defined by (134) satisfies the estimates (132), the proof being the same as in ([Hor] appendix theorem A10). 


\subsection{Proof of Lemma 6.1}

Equation (129) can be transformed into the uncoupled system in $K, L$ :

$$
\begin{gathered}
M_{0}(\theta) \cdot K(\theta)+N_{0}(\theta)=K(\theta+\omega) \cdot\left[N_{-}(\theta) \cdot K(\theta)+M_{-}(\theta)\right], \\
M_{-}(\theta) \cdot L(\theta)+N_{-}(\theta)=L(\theta+\omega) \cdot\left[N_{0}(\theta) \cdot L(\theta)+M_{0}(\theta)\right],
\end{gathered}
$$

and the unknown $M_{0}^{\prime}, M_{-}^{\prime}$ are given by:

$$
\left.\begin{array}{l}
M_{0}^{\prime}(\theta)=N_{0}(\theta) \cdot L(\theta)+M_{0}(\theta), \\
M_{-}^{\prime}(\theta)=N_{-}(\theta) \cdot K(\theta)+M_{-}(\theta) .
\end{array}\right\}
$$

Let us consider Eq. (135) (the other Eq. (136) can be treated similarly) and rewrite it as the operator:

$$
\mathscr{F}(K, W)=-K \circ R_{\omega} \cdot\left[N_{-} \cdot K+M_{-}\right]+M_{0} \cdot K+N_{0},
$$

where

$$
W=\left(M_{0}, M_{-}, N_{0}, N_{-}\right),
$$

defined on the product space corresponding to its arguments, and with values in $C^{\infty}\left[\mathbb{T}^{1},\left(\mathscr{L}\left(E_{-}, E_{0}\right), 0\right)\right]$. We search for $K$ in $C^{\infty}\left[\left(\mathbb{T}^{1},\left(\mathscr{L}\left(E_{-}, E_{0}\right), 0\right)\right)\right.$ satisfying $\mathscr{F}(K, W)=0$. We have $\mathscr{F}\left(0, W_{0}\right) \equiv 0$, with $W_{0}=\left(T_{0}, T_{-}, 0,0\right)$, then we apply to $\mathscr{F}$ the theorem of Hamilton in a neighborhood of $W_{0}$. By Lemma 6.2 all the spaces considered are TFS and the map $\mathscr{F}$ is clearly a tame map since it is defined only with additions and products. Thus we have to show that the partial derivative $\frac{\partial \mathscr{F}}{\partial W}$ $\equiv D_{2} \mathscr{F}$ admits a tame inverse in a neighborhood of $W_{0}$.

By a direct calculation we obtain:

$$
D_{2} \mathscr{F}(K, W) \cdot \Delta K=\left(M_{0}-K \circ R_{\omega} \cdot N_{-}\right) \cdot \Delta K-\Delta K \circ R_{\omega}\left(N_{-} \cdot K+M_{-}\right) .
$$

To invert $D_{2} \mathscr{F}$, we have to solve the linear equation:

$$
D_{2} \mathscr{F}(K, W) \cdot \Delta K=V,
$$

where $V$ is given in $C^{\infty}\left[\left(\mathbb{T}^{1}, \mathscr{L}\left(E_{-}, E_{0}\right)\right)\right.$. In fact we see that:

$$
\left.\begin{array}{l}
\mathscr{M}(\theta)=\left(M_{0}-K \circ R_{\omega} \cdot N_{-}\right)(\theta)=T_{0}+\Theta_{1}(\theta), \\
\left(N_{-} \cdot K+M_{-}\right)(\theta)=T_{-}+\Theta_{2}(\theta),
\end{array}\right\}
$$

where $\Theta_{1}$ and $\Theta_{2}$ are uniformly close to 0 (in $C^{k}$ norm for instance). Now, since the spectrum of $T_{0}$ is on the unit circle and the spectrum of $T_{-}$is inside the open unit disc, we can choose a norm in $E$ such that $\left\|T_{0}\right\|^{-1}$ is as close as we wish to 1 and $\left\|T_{-}\right\|$strictly less than 1 (see [Lan]). As a consequence $\mathscr{M}(\theta)$ is invertible with a norm satisfying:

$$
\left\|\mathscr{M}(\theta)^{-1}\right\|_{0} \leqq 1+\varepsilon, \text { and }\left\|N_{-} \cdot K+M_{-}\right\|_{0} \leqq k+\varepsilon,
$$

where $k<1$ and $\varepsilon$ is small, depending on $\mathscr{T}-\mathscr{T}_{0}$. Now it is clear that the linear operator:

$$
\Delta K \rightarrow \Delta K-\mathscr{M}^{-1}\left[\Delta K \circ R_{\omega}\left(N_{-} \cdot K+M_{-}\right)\right]
$$


is invertible in a neighborhood of $\left(0, W_{0}\right)$ (identity plus a strict contraction). Using standard interpolation inequalities (see for instance [Hor], [Ham]) we can prove easily:

$$
\|\Delta K\|_{m} \leqq C_{m}\left\{1+\|K\|_{m}+\|W\|_{m}\right\}\|V\|_{m}, \quad \forall m \in \mathbb{N}
$$

provided that $\|K\|_{m}+\left\|W-W_{0}\right\|_{m} \leqq \eta, \forall m \in \mathbb{N}$.

This proves that the inverse of the derivative $D_{2} \mathscr{F}$ is a tame map; thus we solve Eq. (135) by the theorem of Hamilton. We end the proof of Lemma 6.1 by proceeding similarly for Eq. (136).

\subsection{Persistence of an Invariant Curve in a Banach Space}

Let $\mathscr{F}_{\mu}$ be a family of maps in the Banach space $E$, satisfying the assumptions of Sect. 2.1, i.e. $\mathscr{F}_{0}(0)=0$ and $D_{Z} \mathscr{F}_{0}(0)=\mathscr{T}_{0}$ has a spectrum separated into $\sum=\left\{\lambda_{0}, \bar{\lambda}_{0}\right.$, $\left.\lambda_{1}, \bar{\lambda}_{1}\right\} \cup \sigma_{-}$, where $\lambda_{j}=e^{2 i \pi \omega_{j}}, j=0,1$, and for every $\lambda \in \sigma_{-},|\lambda|<1$. We showed in Sect. 2.1 how the problem reduces to a 4 dimensional space, but we paid this reduction to the center manifold by loosing the $C^{\infty}$ property.

We now avoid this reduction and use a direct extension of the normalization technique to the infinite dimensional case such as described for vector fields in [El \& al]. We then obtain a family of maps $E_{0} \times E_{-} \rightarrow E_{0} \times E_{-}$, and if we make the same change of coordinates as in Sect. 3.2, we now obtain a family of maps $F_{\mu}$ :

$$
\left.\begin{array}{c}
\mathbb{T}^{1} \times \mathbb{R} \times \mathbb{C} \times E_{-} \rightarrow \mathbb{T}^{1} \times \mathbb{R} \times \mathbb{C} \times E_{-}, \\
F_{\mu}(\theta, x, z, v) \rightarrow(\Theta, X, Z, V), \quad \mu \in\left(\mathbb{R}^{4}, 0\right), \\
\Theta=\theta+\omega_{\mu}+\varepsilon A_{\mu}^{1}\left(x,|z|^{2}\right)+Q_{\mu}^{1}(\theta, x, z, \bar{z}, v), \\
X=(1-\varepsilon) x+\varepsilon A_{\mu}^{2}\left(x,|z|^{2}\right)+Q_{\mu}^{2}(\theta, x, z, \bar{z}, v), \\
Z=e^{2 i \pi\left(\omega_{1}+\sigma\right)}(1+v) z+\varepsilon z A_{\mu}^{3}\left(x,|z|^{2}\right)+Q_{\mu}^{3}(\theta, x, z, \bar{z}, v), \\
V=T_{\mu} v+Q_{\mu}^{4}(\theta, x, z, \bar{z}, v),
\end{array}\right\}
$$

where $Q_{\mu}^{j}(\theta, x, z, \bar{z}, v)=O\left(\varepsilon^{(N / 2)-1}\right), j=1,2,3,4$.

This form is obtained after a scaling: $v=\varepsilon^{q} \cdot v^{\prime}$, with $q \geqq \frac{N}{2}-1$. The linear operator $T_{\mu} \in \mathscr{L}\left(E_{-}\right)$is a deformation, depending smoothly on the parameter $\mu$, of the operator $T_{-}$considered in the previous paragraph.

In this part we sketch the way to extend the persistence result of Sect. 4 by taking into account the contracting part in $E_{-}$. The search of an invariant curve under $F_{\mu}$ in $E$ is performed as in (43), but with an additional unknown function $v(\theta)$ in $C^{\infty}\left[\left(\mathbb{T}^{1}, E_{-}\right)\right.$, which is a TFS by Lemma 6.2. We follow the strategy of Sect. 4 , i.e.:

- Existence of a family of rotated -twisted curves,

- Cancellation of the rotation and the twist parameters.

Expressions occurring in the proof are almost the same as before, so we will not write them again. Operators $\Phi$ and $\mathscr{H}_{t}$ in (49), (50) can easily be extended in order to represent the map in $E$. The main difference between this case and the one considered in Sect. 4 lies in the proof of the $\mathrm{R}-\mathrm{T}$ theorem and more precisely in the 
inversion of the derivative $D_{1} \mathscr{H}_{t}$. This operator is now in the fiber bundle of linear maps in a Banach space over the circle. The sequence of transformations going from (56) to (63) can be rewritten exactly in the same way. At this point we are faced with Eq. (63), correctly reinterpreted with $M^{\prime}(\theta)$ as a linear operator in $\mathscr{L}\left(E^{\prime}\right)$, where $E^{\prime}=\mathbb{R} \times \mathbb{C}^{2} \times E_{\text {_ }}$ close to the constant:

$$
M_{0}^{\prime}=\left(\begin{array}{cc}
M_{0} & 0 \\
0 & T_{\mu}
\end{array}\right)
$$

where the matrix representation corresponds to the product space, and $M_{0}$ is given by (64). Now we apply Lemma 6.1 to $M^{\prime}$, then there exists $\mathbb{H}_{1} \in C^{\infty}\left[\mathbb{T}^{1},\left(\mathscr{L}\left(E^{\prime}\right), \mathrm{Id}\right)\right]$ depending tamely on $M^{\prime}$ such that:

$$
\mathbb{H}_{1}^{-1}(\theta+\omega) \cdot M^{\prime}(\theta) \cdot \mathbb{H}_{1}(\theta)=\left(\begin{array}{cc}
M_{0}^{*}(\theta) & 0 \\
0 & M_{-}^{\prime}(\theta)
\end{array}\right)
$$

Since we have implicitly assumed that $\omega$ satisfies a diophantine condition (46) and $M_{0}^{*}$ satisfies the hypothesis of the quasi-conjugacy theorem A.2, there exists $\left(H, D_{r}, D_{\varphi}\right) \in\left[M_{3 C}^{\infty}\left(\mathbb{T}^{1}, \mathbb{R}\right), \mathrm{Id}\right] \times\left[\operatorname{Diag}_{3}(\mathbb{C}), 0\right]^{2}$ such that:

$$
H^{-1}(\theta+\omega) \cdot\left[\left(M_{0}^{*}(\theta)-D_{\varphi}\right] \cdot H(\theta)=M_{0}+D_{r}=M_{0}^{\prime}, \quad(\text { see }(65)) .\right.
$$

Let us write now: $\mathbb{H}_{2}(\theta)=\left(\begin{array}{cc}H(\theta) & 0 \\ 0 & \mathrm{Id}\end{array}\right) \in C^{\infty}\left[\mathbb{T}^{1},\left(\mathscr{L}\left(E^{\prime}\right), \mathrm{Id}\right)\right]$, then the map: $\mathbb{H}=\mathbb{H}_{1} \cdot \mathbb{H}_{2}$, transforms the operator $M^{\prime}(\theta)$ into:

$$
M^{\prime}(\theta)=\left(\begin{array}{cc}
M_{0}^{\prime} & 0 \\
0 & M_{-}^{\prime}(\theta)
\end{array}\right)
$$

via the quasi-conjugacy relation, provided the twisted parameter $t=t_{0}+\varphi$ is given by (65). At this point we can invert the derivative $D_{1} \mathscr{H}_{t}$, the finite dimensional part of the splitting (125) is exactly given by solving Eq. (67), (68), while the infinite dimensional part is given by solving an equation analogous to (67) but with the linear operator $M_{-}^{\prime}(\theta)$. By (148) the preceding equations are uncoupled and, since $M_{-}^{\prime}(\theta)$ is a strict contraction we can invert the derivative. The resulting inverse is a tame map; this is proved in Sect. 4 for the finite dimensional part, while the argument for the infinite dimensional part is the same as for the proof of Lemma 6.1.

It is then clear that the techniques developed in Sect. 4 work here again with the additional $V$ component, since after decoupling of the linear parts, the linear part with $v$ is a strong contraction. We can then formulate the following:

Complement to the Invariant Curve and Bubbles Theorems (see Sects. 4.5.4 and 5.4). For a family of maps $\mathscr{F}_{\mu}$ in a Banach space E, satisfying the assumptions on the separation of the spectrum of Sect. 2.1, and assumptions of the Bubbles theorem, the same conclusions hold about the families of invariant $\mathbb{T}^{1}$ and bifurcating $\mathbb{T}^{2}$ tori in $E$. 


\section{Appendix: The Quasi-Conjugacy Problem}

\section{A.1. Introduction}

This self contained appendix is devoted to the study of vector difference equations of the form:

$$
G(\theta+\omega)-M(\theta) \cdot G(\theta)=K(\theta), \quad \theta \in \mathbb{T}^{1},
$$

where $G$ and $K$ are $C^{\infty}$-vector valued functions in $\mathbb{R}^{n}, M$ is a given $C^{\infty} n \times n$ matrix valued function, and $\omega$ is a given irrational number in $\mathbb{T}^{1}$. In (A1) the unknown is G.

This type of equation arises naturally as the linear step for proving the existence of $C^{\infty}$ closed curves, diffeomorphic to a circle, with an irrational rotation number, invariant under families of maps in $\mathbb{R}^{n+1}$. The unidimensional case for (A1) is easy to solve, because of the commutativity of the product in $\mathbb{R}$. The method consists in transforming (A1) by the following change of variables:

$$
G(\theta)=H(\theta) \cdot \Gamma(\theta),
$$

where $(\Gamma, H, G)$ are in $C^{\infty}\left(\mathbb{T}^{1}, \mathbb{R}\right)$, in order to obtain an equation for $\Gamma$ of the same type but with a constant instead of $M(\theta)$. For this purpose, the map $H$ which has to be close to identity satisfies:

$$
H^{-1}(\theta+\omega) \cdot M(\theta) \cdot H(\theta)=\text { const. }
$$

When the dimension $n$ is larger than one, Eq. (A3), now with matrices $H$ and $M$, is called a quasi-conjugacy and cannot be solved directly, except for instance in the case of holomorphic matrices (see [Her 83']). Also a special case of Eq. (A3) for $n=2$ is solved in [Los 2], where $M$ is real and close to the constant matrix:

$$
M_{0}=\left(\begin{array}{rr}
1 & 0 \\
0 & -1
\end{array}\right)
$$

In fact this previous result can be generalized easily to the case of $n \times n$ matrices close to a constant diagonal one, with eigenvalues distinct in moduli. Another general result on vector fields is contained in Johnson-Sell [ $\mathrm{Jo}-\mathrm{Se}]$ where strong assumptions are made on the characteristic exponents of $M(\theta)$.

In the present appendix we solve the general case arising in perturbation problems, i.e. when the matrix $M(\theta)$ belongs to a neighborhood of a constant one $M_{0}$. We introduce a small constant matrix to be added to $M$ in order to have the quasi-conjugacy (A3). An analogous result was obtained for a special case by Moser [Mos] for vector fields and $M_{0}$ diagonal with simple pure imaginary eigenvalues.

The constant matrix to be added to $M$ and the one obtained in the right-hand side of (A3) are optimal in the sense that their sum belongs to the so-called "Arnold-Jordan normal form" associated with $M_{0}$ (see [Arn] Sect. 30). The idea is that we want the number of parameters which constitute the constant matrix to be added to $M$, the smallest possible. The most important difference between our result and previous ones is that we do not impose to $M_{0}$ to have distinct eigenvalues. 
The basic idea, which consists in adding some well chosen parameters, goes back to Moser [Mos]. In the same spirit Rüssmann [Russ] introduced a translation parameter in order to prove the existence of an invariant curve for area preserving diffeomorphisms of the annulus, he also used this idea for the study of quasi-periodic Schrödinger equation [ $\mathrm{Ru} 79]$. Following the formulation of Herman [Her], Chenciner [Chen] adapted the method for non-conservative systems, with parameter dependence and a similar adaptation was the introduction of a rotation parameter in [Los 1] for a problem of "curve-doubling bifurcation."

In chapter 4 we introduce 2 parameters: a rotation one and a twist one to prove the persistence of invariant curves. In all these examples the number of parameters introduced is minimal in the general sense described above. This is our motivation to derive a general result which could be used in many other situations.

\section{A.2. Formulation of the Problem}

From now on we only consider the quasi-conjugacy problem (A3), since it is clear that once solved, the resolution of (A1) becomes tractable.

A.2.1. Notations. We use standard notations for spaces of functions: $C^{\infty}\left(\mathbb{T}^{1}, K^{n}\right)$, $K=\mathbb{R}$ or $\mathbb{C}$ is the space of $C^{\infty}$ maps from $\mathbb{T}^{1}$ to $K^{n} ; M_{n}^{\infty}\left(\mathbb{T}^{1}, K\right)$ the space of $n \times n$ matrices whose entries are in $C^{\infty}\left(\mathbb{T}^{1}, K\right) ; M_{n}(K)$ the subspace of constant $n \times n$ matrices; $\operatorname{Diag}_{n}(K)$ the subspace of diagonal $n \times n$ matrices; $\operatorname{Tri}_{n}^{+}(K)$ (respectively $\left.\mathrm{Tri}_{n}^{-}(K)\right)$ the subspace of sup (respectively inf) triangular $n \times n$ matrices.

The topology on $C^{\infty}\left(\mathbb{T}^{1}, K\right)$ is defined by the family $\left\{\|\ldots\|_{k}, k \in \mathbb{N}\right\}$ of $C^{k}$ uniform convergence norms, which defines also the product topology on $C^{\infty}\left(\mathbb{T}^{1}, K^{n}\right)$ and $M_{n}^{\infty}\left(\mathbb{T}^{1}, K\right)$. All these spaces are clearly tame Fréchet spaces (see [Ham]) as product of such spaces. We denote by $(X, A)$ a neighborhood of the set $A$ in the topological space $X$.

A.2.2. The Arnold-Jordan Normal Form. Let $M_{0}$ be in $M_{n}(\mathbb{C})$, the image of the commutator operator (also called "homological" or "adjoint" operator):

$$
\mathscr{A}: M_{n}(\mathbb{C}) \rightarrow M_{n}(\mathbb{C}), \quad \mathscr{A} T \equiv T \cdot M_{0}-M_{0} \cdot T,
$$

is orthogonal to the kernel of $\mathscr{A}^{*}$, where:

$$
\mathscr{A}^{*} T \equiv T \cdot M_{0}^{*}-M_{0}^{*} \cdot T, \quad\left(M_{0}^{*}={ }^{t} \bar{M}_{0}\right),
$$

and where the scalar product in $M_{n}(\mathbb{C})$ is defined by:

$$
\langle A, B\rangle=\operatorname{Tr}\left(A \cdot B^{*}\right), \quad A, B \in M_{n}(\mathbb{C}), \quad(\text { see }[\text { Arn }],[\text { Elp \& al }]) .
$$

Let $M_{0}$ be written in its Jordan form, then $\operatorname{Ker}\left(\mathscr{A}^{*}\right)$ consists in matrices, commuting with $M_{0}^{*}$, which have the form $A+D$, where $D$ is diagonal, proportional to the identity on each subspace corresponding to a Jordan block of $M_{0}$, and where $A$ has a 0 diagonal (in general not triangular). We have the following:

Theorem $\mathrm{A1}$ (see [Arn]). Let $M_{0} \in M_{n}(\mathbb{C})$ be in Jordan form and $M$ be in some neighborhood of $M_{0}$. Then there exists $H \in[G L(n, \mathbb{C})$, Id] (invertible matrices), 
$D \in\left[\operatorname{Diag}_{n}(\mathbb{C}), 0\right], A \in\left[M_{n}(\mathbb{C}), 0\right]$, such that $:$

$$
H^{-1} \cdot M \cdot H=M_{0}+A+D .
$$

The matrices $A$ and $D$ are such that $A+D$ commute with $M_{0}^{*}$, A has a 0 diagonal and $D$ is proportional to the identity on each subspace corresponding to a Jordan block of $M_{0}$. Matrices $A, D$, and $H$ depend analytically on $M$.

A.2.3. The Quasi-Conjugacy Decomposition. We consider now the quasiconjugacy problem (A.3). Let $M_{0}$ be given in $M_{n}(\mathbb{C})$ in Jordan form, and $M(\theta)$ close to $M_{0}$ in $M_{n}^{\infty}\left(\mathbb{T}^{1}, \mathbb{C}\right)$. To prove the quasi-conjugacy result we need to add some parameters as the next theorem states:

Theorem A2. Let $M_{0} \in M_{n}(\mathbb{C})$ in Jordan form and $\omega$ in $\mathbb{T}^{1}$ satisfying the condition:

$$
\exists C>0, \exists \beta \geqq 0 / \forall \frac{p}{q} \in \mathbb{Q},\left|\Omega_{i j}-q \omega-p\right| \geqq \frac{C}{|q|^{1+\beta}},
$$

where $\Omega_{i j}=\frac{1}{2 \pi} \operatorname{Arg}\left(\frac{\lambda_{i}}{\lambda_{j}}\right)$ is defined for every pair $(i, j)$ such that $\left|\lambda_{i}\right|=\left|\lambda_{j}\right|, \lambda_{i}$, $i=1, \ldots, n$ being the eigenvalues of $M_{0}$.

For every matrices $M$ in a small enough neighborhood of $M_{0}$ in $M_{n}^{\infty}\left(\mathbb{T}^{1}, \mathbb{C}\right)$, there exists unique $\left(H, A_{+}, A_{-}, D_{r}, D_{\varphi}, D_{r \psi}\right)$ such that:

$$
\begin{gathered}
H \in\left[M_{n}^{\infty}\left(\mathbb{T}^{1}, \mathbb{C}\right), \operatorname{Id}\right],\left(D_{r}, D_{\varphi}, D_{r \psi}\right) \in\left[\operatorname{Diag}_{n}(\mathbb{C}), 0\right]^{3} \\
\left(A_{+}, A_{-}\right) \in\left[\operatorname{Tri}_{n}^{+}(\mathbb{C}) \times \operatorname{Tri}_{n}^{-}(\mathbb{C}), 0\right], \\
H^{-1}(\theta+\omega) \cdot\left\{M(\theta)-A_{-}-D_{\varphi}\right\} \cdot H(\theta)=M_{0}+A_{+}+D_{r}+D_{r \psi},
\end{gathered}
$$

where

$$
D_{r}=\left(\begin{array}{ccc}
\cdot \cdot \cdot & & 0 \\
0 & \lambda_{i} r_{i} \cdot & \cdot
\end{array}\right), \quad r_{i} \in \mathbb{R}, \quad r_{i}=r_{j} \quad \text { if } \quad \lambda_{i}=\lambda_{j}
$$

and

$$
\begin{aligned}
& D_{\varphi}=\left(\begin{array}{ccc}
\cdot & \cdot & 0 \\
0 & \lambda_{i} m_{i} & 0 \\
0
\end{array}\right), \quad m_{i}=1-e^{-2 i \pi\left(\varphi_{i}-\psi_{\imath}\right)}, \quad \varphi_{i} \in \mathbb{R}, \quad \varphi_{i}=\varphi_{j} \text { if } \lambda_{i}=\lambda_{j}, \\
& D_{r \psi}=\left(\begin{array}{cccc}
\cdot & \cdot & & 0 \\
0 & & \lambda_{i} n_{i} & \cdot \\
\hline
\end{array}\right), \quad n_{i}=1-e^{-2 i \pi \psi_{i}}, \quad \psi_{i} \in \mathbb{R}, \quad \psi_{i}=\psi_{j} \quad \text { if } \quad\left|\lambda_{i}\right|=\left|\lambda_{j}\right|, \\
& \sum_{i ;\left|\lambda_{i}\right|=\left|\lambda_{J}\right|}\left(\varphi_{i}-\psi_{i}\right)=0 \text {, and } A_{+}+A_{-}+D_{r}+D_{\varphi}+D_{r \psi} \text { commutes with } M_{0}^{*} \text {. }
\end{aligned}
$$

The map: $M \rightarrow\left(H, A_{+}, A_{-}, D_{r}, D_{\varphi}, D_{r \psi}\right)$ is tame in the sense of Hamilton [Ham].

Remark 1. (A7) is a mixed diophantine condition between the rotation number $\omega$ and the arguments $\Omega_{i j}$.

Remark 2. The matrix $D_{r}+D_{\varphi}+D_{r \psi}$ has the structure of the diagonal part $D$ of Theorem A1, similarly for $A_{+}+A_{-}$and $A$ of Theorem A1. 
Remark 3. The matrix $D_{r \psi}$ can be chosen as $D_{r \psi}=0$ if we suppress the condition $\sum_{i ;\left|\lambda_{2}\right|=\left|\lambda_{j}\right|}\left(\varphi_{i}-\psi_{i}\right)=0$, when $\left|\lambda_{i}\right|=\left|\lambda_{j}\right|$ for every $i, j$.

Complement for the Real Case. If the matrix $M(\theta)$ is real, i.e. if $M \in M_{n}^{\infty}\left(\mathbb{T}^{1}, \mathbb{R}\right)$, close enough to a real $M_{0}$, there exists a complex change of variable $h$ such that: $h^{-1} \cdot M_{0} \cdot h=M_{0}^{\prime}$ is in Jordan form in $M_{n}(\mathbb{C})$.

If $M_{0}^{\prime}$ satisfies the hypothesis (A7) then the quasi-conjugacy relation (A8) holds for $M$ with real matrices, in particular $D_{r \psi}$ is identically 0 .

Proof of the Complement. The proof is based on the uniqueness property of the quasi-conjugacy. We apply the relation (A8) to the matrix: $h^{-1} \cdot M(\theta) \cdot h$, which is close to $M_{0}^{\prime}$, then we obtain:

$$
\mathscr{H}^{-1}(\theta+\omega) \cdot\left\{M(\theta)-h\left(A_{-}+D_{\varphi}\right) h^{-1}\right\} \cdot \mathscr{H}(\theta)=M_{0}+h\left(A_{+}+D_{r}+D_{r \psi}\right) h^{-1},
$$

with $\mathscr{H}(\theta)=h \cdot H(\theta) \cdot h^{-1}$, then as $M$ and $M_{0}$ are real, by the uniqueness property (consider the equivalent complex conjugate relation) we obtain that: $\mathscr{H}$, $h\left(A_{-}+D_{\varphi}\right) h^{-1}, h\left(A_{+}+D_{r}+D_{r \psi}\right) h^{-1}$ are real matrices. Moreover we have $D_{r \psi}=0$, since for any complex eigenvalue $\lambda_{i}$ there is also $\lambda_{k}=\bar{\lambda}_{i}$ and then $\varphi_{i}=-\varphi_{k}$, hence $\sum_{i ;\left|\lambda_{i}\right|=\left|\lambda_{\jmath}\right|} \varphi_{i}=0$, i.e. $\psi_{i}=0, \forall i$.

Let us explain roughly relationship (A8). We cannot always solve directly the quasi-conjugacy relation (A3) and we need some constants given in (A8) by the parameters appearing in the matrices $A_{-}$and $D_{\varphi}$. We remark that the number of these parameters is governed by the Arnold-Jordan normal form, i.e. by the dimension of a simple subspace of the commutator of $M_{0}^{*}$. This result transforms the quasi-conjugacy problem into the finite codimension condition:

$$
\left.\begin{array}{l}
A_{-}=0 \\
D_{\varphi}=0
\end{array}\right\}
$$

The number of real parameters needed to solve these equations, when $M(\theta)$ depends on parameters, gives the codimension of the problem.

\section{A.3. Proof of the Theorem}

The following proof needs two basic ingredients, the first one is purely algebraic depending on the structure of the matrices and the second one needs some analysis based on the powerful implicit function theorem in Fréchet spaces in the Hamilton formulation [Ham].

Let us write the quasi-conjugacy Eq. (A8) as a functional map:

$$
\begin{gathered}
\mathscr{F}:\left(M_{n}^{\infty}\left(\mathbb{T}^{1}, \mathbb{C}\right)\right)^{2} \times\left(\operatorname{Diag}_{n}(\mathbb{C})\right)^{3} \times \operatorname{Tri}_{n}^{+}(\mathbb{C}) \times \operatorname{Tri}_{n}^{-}(\mathbb{C}) \rightarrow M_{n}^{\infty}\left(\mathbb{T}^{1}, \mathbb{C}\right) \\
\mathscr{F}\left(M, H, D_{r}, D_{\varphi}, D_{r \psi}, A_{+}, A_{-}\right)(\theta) \equiv-M(\theta)+A_{-}+D_{\varphi} \\
+H(\theta+\omega)\left\{M_{0}+A_{+}+D_{r}+D_{r \psi}\right\} H^{-1}(\theta) .
\end{gathered}
$$

We want to solve the equation:

$$
\mathscr{F}(M, X)=0 \quad \text { where } \quad X=\left(H, D_{r}, D_{\varphi}, D_{r \psi}, A_{+}, A_{-}\right),
$$


in a neighborhood of $\left(M_{0}, X_{0}\right)$, where $X_{0}=(\mathrm{Id}, 0,0,0,0,0)$ and $\mathscr{F}\left(M_{0}, X_{0}\right)=0$. The map $\mathscr{F}$ is obviously tame as composed of tame maps, from a tame Fréchet space to another. In order to apply the implicit function theorem of Hamilton, we have to prove that the Gâteaux partial derivative:

$$
\frac{\partial \mathscr{F}}{\partial X} \equiv D_{2} \mathscr{F},
$$

admits a tame inverse in a neighborhood of $\left(M_{0}, X_{0}\right)$.

The direct calculation of this derivative gives:

$$
\begin{aligned}
D_{2} \mathscr{F}(M, X)(\Delta X)= & \Delta A_{-}+\Delta D_{\varphi}+\Delta H(\theta+\omega) \cdot\left\{M_{0}+A_{+}+D_{r}+D_{r \psi}\right\} \cdot H^{-1}(\theta) \\
& +H(\theta+\omega) \cdot\left\{\Delta A_{+}+\Delta D_{r}+\Delta D_{r \psi}\right\} \cdot H^{-1}(\theta) \\
& -H(\theta+\omega) \cdot\left\{M_{0}+A_{+}+D_{r}+D_{r \psi}\right\} \cdot H^{-1}(\theta) \Delta H(\theta) H^{-1}(\theta) .
\end{aligned}
$$

Proving the existence of an inverse for $D_{2} \mathscr{F}$ consists in solving, for any $N \in M_{n}^{\infty}\left(\mathbb{T}^{1}, \mathbb{C}\right)$, the following linear equation in $\Delta X$ :

$$
D_{2} \mathscr{F}(M, X)(\Delta X)=N,
$$

for any $(M, X)$ in a neighborhood of $\left(M_{0}, X_{0}\right)$.

Defining new notations:

$$
\left\{\begin{array}{l}
\Delta H^{\prime}(\theta)=H^{-1}(\theta) \Delta H(\theta) \\
N^{\prime}(\theta)=H^{-1}(\theta+\omega) \cdot N(\theta) \cdot H(\theta),
\end{array}\right.
$$

Eq. (A10) becomes:

$$
\begin{aligned}
& \Delta H^{\prime}(\theta+\omega)\left\{M_{0}+A_{+}+D_{r}+D_{r \psi}\right\}-\left\{M_{0}+A_{+}+D_{r}+D_{r \psi}\right\} \Delta H^{\prime}(\theta)+\Delta A_{+}+\Delta D_{r} \\
& \quad+\Delta D_{r \psi}+H^{-1}(\theta+\omega) \cdot\left\{\Delta A_{-}+\Delta D_{\varphi}\right\} \cdot H(\theta)=N^{\prime}(\theta) .
\end{aligned}
$$

This relation looks like a commutator operator which we shall solve by a Fourier series method. Let us define the following series:

$$
\begin{aligned}
\Delta H^{\prime}(\theta) & =\sum_{p \in \mathbb{Z}} e^{2 i \pi p \theta} \cdot \Delta H_{p}^{\prime}, \quad \Delta H_{p}^{\prime} \in M_{n}(\mathbb{C}), \\
N^{\prime}(\theta) & =\sum_{p \in \mathbb{Z}} e^{2 i \pi p \theta} \cdot N_{p}^{\prime}, \quad N_{p}^{\prime} \in M_{n}(\mathbb{C}) .
\end{aligned}
$$

Then the constant part of Eq. (A11) is given by:

$$
\begin{gathered}
\Delta H_{0}^{\prime}\left\{M_{0}+A_{+}+D_{r}+D_{r \psi}\right\}-\left\{M_{0}+A_{+}+D_{r}+D_{r \psi}\right\} \Delta H_{0}^{\prime} \\
+\Delta A_{+}+\Delta D_{r}+\Delta D_{r \psi}+\left\{\Delta A_{-}+\Delta D_{\varphi}\right\}_{0}^{\prime}=N_{0}^{\prime},
\end{gathered}
$$

the remaining part, i.e. terms in $e^{2 i \pi p \theta}$, is given by:

$$
\begin{gathered}
e^{2 i \pi p \omega} \cdot \Delta H_{p}^{\prime}\left\{M_{0}+A_{+}+D_{r}+D_{r \psi}\right\}-\left\{M_{0}+A_{+}+D_{r}+D_{r \psi}\right\} \Delta H_{p}^{\prime} \\
+\left\{\Delta A_{-}+\Delta D_{\varphi}\right\}_{p}^{\prime}=\mathrm{N}_{p}^{\prime} .
\end{gathered}
$$


The end of this paragraph is devoted to the solving of these two equations, which is the main point of this appendix, and proving that the resulting series $\Delta H^{\prime}$ actually converge.

A.3.1. Solution to the Mean Value Equation. Equation (A12) makes appear a commutator of the same kind as the one occurring while proving Theorem A1. Let us define the following linear operator in $M_{n}(\mathbb{C})$ :

$$
\mathscr{A}_{0}: \Delta H_{0}^{\prime} \rightarrow \Delta H_{0}^{\prime} \cdot M_{0}-M_{0} \cdot \Delta H_{0}^{\prime},
$$

and

$$
\mathscr{A}_{\varepsilon}: \Delta H_{0}^{\prime} \rightarrow \Delta H_{0}^{\prime} \cdot\left(A_{+}+D_{r}+D_{r \psi}\right)-\left(A_{+}+D_{r}+D_{r \psi}\right) \cdot \Delta H_{0}^{\prime},
$$

the index $\varepsilon$ is only used to indicate the smallness of the norm of $\mathscr{A}_{\varepsilon}$.

Equation (A12) now reads:

$$
\mathscr{A}_{0} \cdot \Delta H_{0}^{\prime}+\mathscr{A}_{\varepsilon} \cdot \Delta H_{0}^{\prime}=N_{0}^{\prime}-F_{0},
$$

where $N_{0}^{\prime}$ is given in $M_{n}(\mathbb{C})$. We look for $\Delta H_{0}^{\prime}$ and the simplest $F_{0}$ possible. Let us denote by $\Pi_{0}$ the orthogonal projection on the kernel of $\mathscr{A}_{0}^{*}$. Since $\Delta D_{r}+\Delta D_{\varphi}$ $+\Delta D_{r \psi}+\Delta A_{+}+\Delta A_{-}$is in $\operatorname{Ker}\left(\mathscr{A}_{0}^{*}\right)$ (as well as $D_{r}+D_{\varphi}+D_{r \psi}+A_{+}+A_{-}$) it respects the decomposition indicated at Theorem A2 and it is then clear that $F_{0}$ cannot be chosen in $\operatorname{Ker}\left(\mathscr{A}_{0}^{*}\right)$ because in general:

$$
\left(\mathrm{Id}-\Pi_{0}\right)\left\{\left[\Delta A_{-}+\Delta D_{\varphi}\right]^{\prime}\right\}_{0} \neq 0 .
$$

By using $\Pi_{0}$ we decompose now Eq. (A14) which becomes the following system:

$$
\begin{gathered}
{\left[\mathscr{A}_{0}+\left(\mathrm{Id}-\Pi_{0}\right) \mathscr{A}_{\varepsilon}\right] \Delta H_{0}^{\prime}+\left(\mathrm{Id}-\Pi_{0}\right)\left\{\left[\Delta A_{-}+\Delta D_{\varphi}\right]^{\prime}\right\}_{0}=\left(\mathrm{Id}-\Pi_{0}\right) N_{0}^{\prime},} \\
\Pi_{0} \mathscr{A}_{\varepsilon} \Delta H_{0}^{\prime}+\Delta D_{r}+\Delta D_{r \psi}+\Delta A_{+}+\Pi_{0}\left\{\left[\Delta A_{-}+\Delta D_{\varphi}\right]^{\prime}\right\}_{0}=\Pi_{0} N_{0}^{\prime} .
\end{gathered}
$$

Let us define a complementary space to $\operatorname{Ker}\left(\mathscr{A}_{0}\right)$ in $M_{n}(\mathbb{C})$, then $\mathscr{A}_{0}$ is invertible from this subspace to $\operatorname{Im}\left(\mathscr{A}_{0}\right)$. Now observing that:

$$
(\ldots)^{\prime}=H^{-1}(\theta+\omega)(\ldots) H(\theta),
$$

we write $H=\mathrm{Id}+h, h$ being uniformly close to 0 , so we have:

$$
\left(\Delta A_{-}+\Delta D_{\varphi}\right)^{\prime}=[\operatorname{Id}+O(h)]\left(\Delta A_{-}+\Delta D_{\varphi}\right) .
$$

It is then clear that the linear operator on the left-hand side of (A15-16) which acts on $\Delta H_{0}^{\prime}, \Delta D_{r}+\Delta D_{r \psi}+\Delta A_{+}, \Delta A_{-}+\Delta D_{\varphi}$, is a small perturbation of an invertible operator, hence it is invertible for $\|h\|$ and $\left\|\mathscr{A}_{\varepsilon}\right\|$ small enough (in $C^{0}$ norm).

Remark 1. We can observe that the solution of (A15-16) verifies $\Delta D_{r}+\Delta D_{r \psi}+\Delta A_{+}$ $+\Delta A_{-}+\Delta D_{\varphi}=\Pi_{0} N_{0}^{\prime}+$ h.o.t., where h.o.t. means terms of order $\|h\|$ and $\left\|\mathscr{A}_{\varepsilon}\right\|$.

Remark 2. The choice of $F_{0}(\mathrm{~A} 14)$ is in fact governed by the semi-continuity property of the codimension of the image for perturbations of linear operators [Kato]. This means that the codimension might only become smaller in the neighborhood of $h=0$. Then assuming $F_{0}$ in a subspace having the dimension of $\operatorname{Ker}\left(\mathscr{A}_{0}^{*}\right)$ insures us a regular dependency as $h \rightarrow 0$ (i.e. $M \rightarrow M_{0}$ ). 
Then we have solved Eq. (A12) with respect to $\Delta H_{0}^{\prime}, \Delta D_{r}, \Delta D_{\varphi}, \Delta D_{r \psi}, \Delta A_{+}$, and $\Delta A_{-}$by Eqs. (A15), (A16) above.

A.3.2. Solution of the Equation (A13), $|n| \geqq 1$. Let us define the matrices: $M_{0}+D_{r}$ $+A_{+}+D_{r \psi}=\left\{\mu_{i j}\right\}_{i, j=1, \ldots, n}, \quad\left(\mu_{i i}=\mu_{i}\right)$ in $\operatorname{Tri}_{n}^{+}(\mathbb{C})$, and $\Delta H_{p}^{\prime}=\left\{h_{i j}\right\}_{i, j=1, \ldots, n}$, in $M_{n}(\mathbb{C})$.

Equation (A13) now reads:

$$
\left(e^{2 i \pi p \omega} \mu_{j}-\mu_{i}\right) h_{i j}+\sum_{k<j} \mu_{k j} \cdot e^{2 i \pi p \omega} \cdot h_{i k}-\sum_{k>i} \mu_{i k} \cdot h_{k j}=r_{i j},
$$

where: $\left\{r_{i j}\right\}_{i, j=1, \ldots, n}=N_{p}^{\prime}-\left[\left(\Delta D_{\varphi}+\Delta A_{-}\right)^{\prime}\right]_{p}$, which is well defined by the point A.3.1.

The system (A18) is triangular in $\mathbb{C}^{n^{2}}$ as can be seen in denoting the components of the vector $\left\{h_{i j}\right\}$ by:

$$
h=\left(h_{1, n} ; h_{1, n-1} ; \ldots ; h_{11} ; h_{2, n} ; \ldots ; h_{2,1} ; \ldots ; h_{i, n} ; \ldots ; h_{i, 1} ; \ldots ; h_{n, 1}\right) .
$$

The corresponding matrix for the system (A18) is triangular, as it is clear by the following $(i, j)$-row:

$$
\begin{aligned}
& \left\{\left[0 ; \ldots ; 0 ;\left(\mu_{j} e^{2 i \pi p \omega}-\mu_{i}\right) ; e^{2 i \pi p \omega} \cdot \mu_{j-1 ; j} ; \ldots ; \mu_{1 ; j} e^{2 i \pi p \omega} ;\right.\right. \\
& .(i ; j) \ldots \ldots \ldots(i ; j-1) \ldots \ldots \ldots \ldots(i ; 1) \ldots \\
& \left.\begin{array}{r}
\left.\ldots 0 ; \ldots ; 0 ;-\mu_{i ; i+1} ; \ldots ;-\mu_{i ; n} ; 0 ; \ldots ; 0\right] \\
\ldots \ldots \ldots \ldots(1+1 ; j) \ldots \ldots(n ; j) \ldots \ldots \ldots \ldots . .
\end{array}\right\} .
\end{aligned}
$$

From this triangular structure the system is obviously invertible, for each integer $p$, since by the condition (A7): $\left(e^{2 i \pi p \omega} \mu_{j}-\mu_{i}\right) \neq 0, \forall i, j=1, \ldots, n$. Furthermore the inverse of this system is bounded by:

$$
K \cdot\left[\prod_{i ; j=1}^{n}\left(e^{2 i \pi p \omega} \mu_{j}-\mu_{i}\right)\right]^{-1}, \quad K>0 \text { constant } .
$$

This enables us to prove convergence of the Fourier series: $\sum_{p \in \mathbb{Z}} e^{2 i \pi p \theta} \cdot \Delta H_{p}^{\prime}$, using condition (A7) to bound each term $\Delta H_{p}^{\prime}$, as it is the case for standard difference equations [Her], these estimates implies a loss of differentiability for $\Delta H^{\prime}$, as in the standard case. This loss depends on the diophantine constant $\beta$ and on the integer $L$ which is the number of pairs $(i, j)$ such that $\left|\lambda_{i}\right|=\left|\lambda_{j}\right|\left(L \leqq n^{2}\right)$. The bound (A19) is the worst one corresponding to triangular matrices, while for diagonal one it is replaced by:

$$
K \cdot \operatorname{Sup}\left\{\left(e^{2 i \pi p \omega} \mu_{j}-\mu_{i}\right)^{-1} ; i, j=1, \ldots, n\right\},
$$

the loss of differentiability is then only: $1+\beta^{\prime}, \beta^{\prime}>\beta$.

Nevertheless for all cases the precise estimate is of the form:

$$
\left.\begin{array}{l}
\exists R \in \mathbb{N} ; R \geqq L(1+\beta) \text { or } R \geqq(1+\beta) / \forall m \in \mathbb{N} ; \exists C(m)>0 \text { such that: } \\
\left\{\left\|\Delta H^{\prime}\right\|_{m}+|\Delta Y|\right\} \leqq C(m)\left\{\left\|N^{\prime}\right\|_{m+R}+\|H-\mathrm{Id}\|_{m+R}+|Y|\right\},
\end{array}\right\}
$$

where $Y$ is defined by the 5 last components of $X$ [see (A9)].

We remark at this point that the diophantine condition (A7) is uniform in $\beta$, which means that for any pair $(i, j)$ we have the same $\beta$. It is clear that some more 
precise conditions replacing (A7) might be defined. In our case condition (A7) is sufficient. Estimate (A20) implies that the map:

$$
\left(D_{2} \mathscr{F}\right)^{-1}:(N, M, X) \rightarrow \Delta X=\left(\Delta H, \Delta D_{r}, \Delta D_{\varphi}, \Delta D_{r \psi}, \Delta A_{+}, \Delta A_{-}\right)
$$

is tame, so we can complete the proof of Theorem 2 using the theorem of Hamilton [Ham].

\section{A.4. Some Applications}

In order to illustrate the preceding result we give here some applications, one of them being the precise case we need for the main part of the paper.

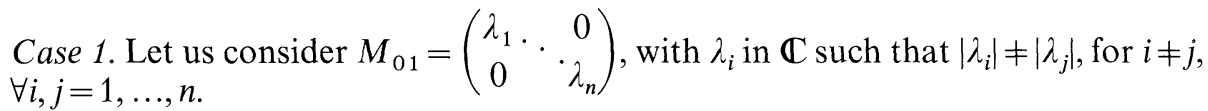

In this case the quasi-conjugacy equation admits a solution, i.e. there is no matrix to be added on the left-hand side of (A3) and the constant matrix on the right-hand side is of the form:

$$
M_{01}^{\prime}=M_{01}+D_{r}+D_{r \psi}=\left(\begin{array}{ccc}
\lambda_{1}\left(1+a_{1}\right) & 0 \\
0 & \ddots_{n}\left(1+a_{n}\right)
\end{array}\right), \quad a_{i} \in(\mathbb{C}, 0), \quad i=1, \ldots, n .
$$

The condition (A7) is reduced to the standard diophantine condition on $\omega$.

Corollary 1. For every $M$ close to $M_{01}$ in $M_{n}^{\infty}\left(\mathbb{T}^{1}, \mathbb{C}\right)$ and $\omega$ satisfying a diophantine condition, there exists $a_{i} \in(\mathbb{C}, 0), i=1, \ldots, n$ and $H \in\left[M_{n}^{\infty}\left(\mathbb{T}^{1}, \mathbb{C}\right)\right.$, Id $]$ such that:

$$
H^{-1}(\theta+\omega) \cdot M(\theta) \cdot H(\theta)=M_{01}^{\prime}=\left(\begin{array}{ccc}
\lambda_{1}\left(1+a_{1}\right) & 0 \\
0 & \ddots & \lambda_{n}\left(1+a_{n}\right)
\end{array}\right) \text {. }
$$

Case 2. Let $M_{02}=\left(\begin{array}{rr}1 & 0 \\ 0 & -1\end{array}\right)$, we consider $M$ close to $M_{02}$ in $M_{2}^{\infty}\left(\mathbb{T}^{1}, \mathbb{R}\right)$. The quasi-conjugacy problem admits a solution with: $A_{+}=A_{-}=D_{\varphi}=D_{r \psi}=0$, and:

$$
M_{02}+D_{r}=\left(\begin{array}{cc}
1+\varrho & 0 \\
0 & -(1+\chi)
\end{array}\right)=M_{02}^{\prime} \text {. The condition (A7) is a standard dio- }
$$
phantine condition on $\omega$.

Corollary 2. Let $M$ close to $M_{02}$ in $M_{2}^{\infty}\left(\mathbb{T}^{1}, \mathbb{R}\right)$ and $\omega$ satisfying a diophantine condition, then there exists $(\varrho, \chi) \in\left(\mathbb{R}^{2}, 0\right)$ and $H \in\left[M_{2}^{\infty}\left(\mathbb{T}^{1}, \mathbb{R}\right), \operatorname{Id}\right]$ such that: $H^{-1}(\theta+\omega) \cdot M(\theta) \cdot H(\theta)=M_{02}^{\prime}$.

This result is proved by a direct method in [Los 2].

Case 3. Let us consider $M_{03}=\left(\begin{array}{ll}1 & 1 \\ 0 & 1\end{array}\right)$, matrices $A+D$ of Theorem A1 have the form: $A+D=\left(\begin{array}{ll}a & 0 \\ b & a\end{array}\right),(a, b) \in \mathbb{R}^{2}$, this means that $D_{r}=\left(\begin{array}{ll}a & 0 \\ 0 & a\end{array}\right)$ and $A_{-}=\left(\begin{array}{ll}0 & 0 \\ b & 0\end{array}\right)$, the other constant matrices appearing in (A8) being identically zero. The condition (A7) is the standard diophantine one. 
Corollary 3. Let $M \in\left[M_{2}^{\infty}\left(\mathbb{T}^{1}, \mathbb{R}\right), M_{03}\right]$ and $\omega$ satisfy a diophantine condition. Then there exists $(a, b) \in\left(\mathbb{R}^{2}, 0\right)$ and $H \in\left[M_{2}^{\infty}\left(\mathbb{T}^{1}, \mathbb{R}\right)\right.$, Id $]$ such that:

$$
H^{-1}(\theta+\omega) \cdot\left[M(\theta)-\left(\begin{array}{ll}
0 & 0 \\
b & 0
\end{array}\right)\right] \cdot H(\theta)=\left(\begin{array}{cc}
1+a & 1 \\
0 & 1+a
\end{array}\right) \text {. }
$$

We have then to "translate" the matrix $M$ by $\left(\begin{array}{ll}0 & 0 \\ b & 0\end{array}\right)$ to get the quasi-conjugacy to a constant triangular matrix. In order to obtain a strict quasi-conjugacy we have to solve: $b(M)=0$, so this is a codimension one problem.

Case 4. We consider now the matrix $M_{04}=\left(\begin{array}{cc}\lambda_{0} & 0 \\ 0 & \bar{\lambda}_{0}\end{array}\right)$ in $M_{2 C}(\mathbb{R})$, the complexified space of $M_{2}(\mathbb{R})$ in $M_{2}(\mathbb{C}), \lambda_{0}=e^{2 i \pi \Omega_{0}}, \Omega_{0} \neq 0,1 / 2$. By Theorem A1 in the real case we have:

$A+D=\left(\begin{array}{cc}c & 0 \\ 0 & \bar{c}\end{array}\right)$, thus $A_{+}=A_{-}=0$ and:

$D_{r}=\left(\begin{array}{cc}\alpha \lambda_{0} & 0 \\ 0 & \alpha \bar{\lambda}_{0}\end{array}\right), \alpha \in(\mathbb{R}, 0) \quad$ and $\quad D_{\varphi}=\left(\begin{array}{cc}\left(1-e^{2 i \pi \varphi}\right) \lambda_{0} & 0 \\ 0 & \left(1-e^{-2 i \pi \varphi}\right) \bar{\lambda}_{0}\end{array}\right), \varphi \in(\mathbb{R}, 0)$.

The condition (A7) is now:

$$
\left.\begin{array}{l}
\left|2 \Omega_{0}-q \omega-p\right| \geqq \frac{C}{|q|^{1+\beta}} \\
|q \omega-p| \geqq \frac{C}{|q|^{1+\beta}},
\end{array}\right\}
$$

and we have:

Corollary 4. Let $M \in\left[M_{2 C}^{\infty}\left(\mathbb{T}^{1}, \mathbb{R}\right), M_{04}\right]$, the complexified space of matrices of real operators, and let $\Omega_{0}$ and $\omega$ satisfy the condition (A22), then there exists $(\alpha, \varphi) \in\left(\mathbb{R}^{2}, 0\right)$, and $H \in\left[M_{2 C}^{\infty}\left(\mathbb{T}^{1}, \mathbb{R}\right)\right.$, Id] such that:

$$
H^{-1}(\theta+\omega) \cdot\left[M(\theta)-D_{\varphi}\right] \cdot H(\theta)=\left(\begin{array}{cc}
(1+\alpha) \lambda_{0} & 0 \\
0 & (1+\alpha) \bar{\lambda}_{0}
\end{array}\right)
$$

We have then to "twist" the matrix $M$ to get the quasi-conjugacy. This problem is, as the preceding one, of codimension one since we should have to solve $\varphi(M)=0$ in order to obtain a quasi-conjugacy.

Case 5. We consider now the case needed for the bifurcation problem studied in this paper; let

$$
M_{05}=\left(\begin{array}{ccc}
1 & 0 & 0 \\
0 & \lambda_{0} & 0 \\
0 & 0 & \bar{\lambda}_{0}
\end{array}\right)
$$


in $M_{3 C}(\mathbb{R})$, with $\lambda_{0}=e^{2 i \pi \Omega_{0}} \neq \pm 1$. Matrices $A, D$ of Theorem 1 have the form:

$$
\left.\begin{array}{rl}
A+D=\left(\begin{array}{lll}
a & 0 & 0 \\
0 & b & 0 \\
0 & 0 & \bar{b}
\end{array}\right),(a, b) \in(\mathbb{R} \times \mathbb{C}, 0), \text { then } A_{+}=A_{-}=0, \text { and: } \\
D_{r}=\left(\begin{array}{ccc}
a & 0 & 0 \\
0 & \lambda_{0} \alpha & 0 \\
0 & 0 & \bar{\lambda}_{0} \alpha
\end{array}\right) ; \quad(a, \alpha) \in\left(\mathbb{R}^{2}, 0\right) ; \\
D_{\varphi}=\left(\begin{array}{ccc}
0 & 0 & 0 \\
0 & \left(1-e^{2 i \pi \varphi}\right) \lambda_{0} & 0 \\
0 & 0 & \left(1-e^{-2 i \pi \varphi}\right) \bar{\lambda}_{0}
\end{array}\right) ; \quad \varphi \in(\mathbb{R}, 0) ;
\end{array}\right\}
$$

The condition (A7) now reads:

$$
\left.\begin{array}{l}
\left|2 \Omega_{0}-q \omega-p\right| \geqq \frac{C}{|q|^{1+\beta}} \\
\left|\Omega_{0}-q \omega-p\right| \geqq \frac{C}{|q|^{1+\beta}} \\
|q \omega-p| \geqq \frac{C}{|q|^{1+\beta}},
\end{array}\right\}
$$

and we have:

Corollary 5. Let $M \in\left[M_{3 C}^{\infty}\left(\mathbb{T}^{1}, \mathbb{R}\right), M_{05}\right]$, the complexified space of matrices of real operators, and let $\Omega_{0}$ and $\omega$ satisfy the condition (A25), then there exists $(a, \alpha, \varphi) \in\left(\mathbb{R}^{3}, 0\right)$, and $H \in\left[M_{3 C}^{\infty}\left(\mathbb{T}^{1}, \mathbb{R}\right), \mathrm{Id}\right]$ such that :

$$
H^{-1}(\theta+\omega) \cdot\left[\left(M(\theta)-D_{\varphi}\right] \cdot H(\theta)=M_{05}+D_{r},\right.
$$

where $D_{r}, D_{\varphi}$ are given by (A24).

We remark immediately that the second condition (A25) can be suppressed since it is implied by the first one. Relation (A26) is again a twisted quasiconjugacy of codimension one.

Theorem A2 has a wide range of applicability for problems of bifurcations of invariant tori for which it has been designed. The generalization to $\mathbb{T}^{n}$ instead of $\mathbb{T}^{1}$ is straightforward.

Acknowledgements. We thank Alain Chenciner for fruitful discussions and remarks on this work.

\section{References}

[Arn] Arnold, V.I.: Chapitres supplémentaires de la théorie des équations différentielles. Moscou: MIR 1980

[Bel] Belitskii, G.R.: Normal forms relative to a filtering action of a group. Trudy Mosk. Mat. Obchestva 40 (1979)

[Bost] Bost, J.B.: Tores invariants des systèmes dynamiques hamiltoniens. Séminaire Bourbaki 1984-1985 no. 639 
[B.H.T.] Broer, H.W., Huitema, G.B., Takens, F.: Unfoldings of quasi-periodic tori. Preprint (Univ. of Groningen)

[Bra-B] Braaksma, B.L.J., Broer, H.W.: On a quasi-periodic Hopf bifurcation. Ann. Inst. Henri Poincaré. Analyse non linéaire 4, 2, 115-168 (1987)

[Ch-Io] Chenciner, A., Iooss, G.: Bifurcation de tores invariants. Arch. Rat. Mech. Anal. 69, 3, 109-198 (1979); and Persistance et bifurcation de tores invariants. Arch. Rat. Mech. Anal. 71, 4, 301-306 (1979)

[Chen] Chenciner, A.: Bifurcation de point fixes elliptiques I, Courbes invariantes. Publ. Math. IHES 61, 67-127 (1985)

[Dou] Douady, R.: Applications du théorème des tores invariants, Thèse 3ème cycle, Paris VII (1982)

[El\&al] Elphick, C., Tirapegui, E., Brachet, M., Coullet, P., Iooss, G.: A simple global characterization for normal forms of singular vector fields. Physica D 29, 95-127 (1987)

[Go-Be] Gollub, J.P., Benson, S.V.: Many routes to turbulent convection. J. Fluid. Mech. 100, 3, 449 (1980)

[GRS] Gorman, M., Reith, L.A., Swinney, H.L.: Modulation patterns, multiple frequencies, and other phenomena in circular Couette flow. Nonlinear dynamics. Ann. N.Y. Acad. Sci. 357, 10-21, R. Helleman (ed.) (1980)

[Gu-Ho] Guckenheimer, J., Holmes, P.: Nonlinear oscillations, dynamical systems, bifurcations of vector fields. Appl. Math. Sci. Vol. 42. Berlin, Heidelberg, New York: Springer 1983

[Ham 74] Hamilton, R.S.: The inverse function theorem of Nash-Moser. Preprint Cornell University (1974)

[Ham] Hamilton, R.S.: The inverse function theorem of Nash-Moser. Bull. Am. Math. Soc. 7, 1, 65-222 (1982)

[Her 79] Herman, M.: Sur la conjugaison différentiable des difféomorphismes du cercle à des rotations. Pub. Math. IHES 49, 5-233 (1979)

[Her 83] Herman, M.: Sur les courbes invariantes par les difféomorphismes de l'anneau I. Astérisque 103-104 (1983)

[Her 83'] Herman, M.: Une méthode pour minorer les exposants de Lyapunov et quelques exemples montrant le caractère local d'un théorème d'Arnold et de Moser sur le tore de dimension 2. Comment Math. Helv. 58, 453-502 (1983)

[Her] Herman, M.: Sur les courbes invariantes par les difféomorphismes de l'anneau (to appear in Astérisque)

[Hor] Hormander, L.: The boundary problem of physical geodesy. Arch. Rat. Mech. Anal. 62, 1-52 (1976)

[Io-La] Iooss, G., Langford, W.F.: Conjectures on the route to turbulence via bifurcations. Nonlinear dynamics. Ann. N.Y. Acad. Sci. 357, 489-505, R. Helleman (ed.) (1980)

[Io 79] Iooss, G.: Bifurcation of maps and applications. North Holland Math. Studies, Vol. 36. Amsterdam: North Holland 1979

[Io 81] Iooss, G.: Bifurcations élémentaires-successions et interactions. Nonlinear phenomena in Chemical dynamics. Vidal-Pacault (ed.) pp. 71-78 (1981)

[Io 84] Iooss, G.: Some codimension two bifurcations for maps, leading to chaos. Chaos and statistical methods. Kuramoto (ed.), pp. 136-142. Berlin, Heidelberg, New York: Springer 1984

[Io 87] Iooss, G.: Formes normales d'applications. Caractérisation globale et méthode de calcul. Preprint no. 132, Univ. Nice (1987)

[Jo-Se] Johnson, R., Sell, G.: Smoothness of spectral subbundles and reductibility of quasiperiodic linear differential systems. J. Differ. Equations 41, 262-288 (1981)

[Jo-Ze] Jost, R., Zehnder, E.: A generalization of the Hopf bifurcation theorem. Helv. Phys. Acta 45, 258-276 (1972)

[Kato] Kato, T.: Perturbation theory for linear operators. Berlin, Heidelberg, New York: Springer 1966 
[La-Io] Langford, W.F., Iooss, G.: Interactions of Hopf and pitchfork bifurcations. Bifurcations problems and their numerical solution. Mittelmann, Weber (ed.) ISNM, Vol. 54, pp. 103-134. Basel: Birkhäuser 1980

[Lan] Lanford, O.E.: Bifurcation of periodic solutions into invariant tori: The work of Ruelle and Takens. Lecture Notes in Mathematics, Vol. 322, p. 159-192. Berlin, Heidelberg, New York: Springer 1973

[Los 1] Los, J.E.: Phénomènes de petits diviseurs dans les dédoublements de courbes invariantes. Ann. Inst. Henri Poincaré, Analyse non linéaire 5, 1, 37-95 (1988)

[Los 2] Los, J.E.: Non normally hyperbolic invariant curves for maps in $\mathbb{R}^{3}$ and doubling bifurcation. Nonlinearity (to appear)

[Ma-Cr] Marsden, J., McCracken, M.: The Hopf bifurcation and its applications. Appl. Math. Sci., vol. 19. Berlin, Heidelberg, New York: Springer 1976

[Mos] Moser, J.: Convergent series expansion for quasi-periodic motion. Math. Ann. 169, 136-176 (1967)

[Ru-Ta] Ruelle, D., Takens, F.: On the nature of turbulence. Commun. Math. Phys. 20,167-192 (1971)

[Ruel] Ruelle, D.: Bifurcations in the presence of a symmetry group. Arch. Rat. Mech. Anal. 51, 136-152 (1973)

[Russ] Rüssmann, H.: Über invariante Kurven differenzierbarer Abbildungen eines Kreisrings. Nach. Akad. Wiss. Göttingen Math. Phys. K1. II, 67-105 (1970)

[Ru 79] Rüssmann, H.: On the one dimensional Schrödinger equation with a quasi-periodic potential. Ann. N.Y. Acad. Sci. 357, 90-107 (1980)

[Sch] Scheurle, J.: Bifurcation of quasi-periodic solutions from quilibrium points of reversible dynamical systems. Arch. Rat. Mech. Anal. 97, 2, 103-139 (1987)

[Sell] Sell, G.R.: Bifurcation of higher dimensional tori. Arch. Rat. Mech. Anal. 69, 3, 199-230 (1979)

[Si-Mo] Siegel, C.L., Moser, J.: Lectures on celestial mechanics. Berlin, Heidelberg, New York: Springer 1971

[Yoc] Yoccoz, J.C.: Conjugaison différentiable des difféomorphismes du cercle dont le nombre de rotation vérifie une condition diophantienne. Ann. Sci. ENS 17, 333-359 (1984)

[Zehn] Zehnder, E.: Generalized implicit function theorems with application to small divisor problems I. Commun. Pure Appl. Math. 28, 91-140 (1975)

Communicated by J.-P. Eckmann

Received January 19, 1988 